\title{
Performance Analysis of Full-Duplex Relay-Aided NOMA Systems Using Partial Relay Selection
}

\author{
Anderson Tregancini, Edgar Eduardo Benitez Olivo, Member, IEEE, Diana Pamela Moya Osorio, Member, IEEE, \\ Carlos H. M. de Lima, Member, IEEE, and Hirley Alves, Member, IEEE
}

\begin{abstract}
Capitalizing on the benefits of non-orthogonal multiple access (NOMA) and full-duplex relaying as key technologies to boost spectral efficiency in the next generation of wireless communications, herein we investigate the performance of a cooperative network in which a source communicates with two destinations via one node selected from a set of full-duplex amplify-and-forward relays. For this purpose, a power-domain NOMA scheme is used to transmit information from the source to the destinations, and partial relay selection is performed to choose the relay based on the channel state information of the first hops. The system performance is characterized in terms of both the outage probability and the ergodic capacity, for which, exact analytical expressions are derived in integral form. In addition, to reduce the computational complexity of the obtained analytical results, closed-form expressions are derived from lower-bound, approximate, and asymptotic analyses. From these analytical expressions, the impact on the system performance of the number of relays, the power allocation factor between the NOMA destinations, and the residual self-interference at full-duplex relays is assessed. The correctness of our analyses is validated by Monte Carlo simulations, and a comparison with the half-duplex relay-aided NOMA system counterpart is also provided.
\end{abstract}

Index Terms-amplify-and-forward, ergodic capacity, fullduplex relaying, NOMA, outage probability, partial relay selection.

\section{INTRODUCTION}

$\mathbf{T}$ HE fifth-generation $(5 \mathrm{G})$ of wireless networks is expected to be a major revolution in wireless communications, so that stringent requirements must be met, such as extremely high data throughput, very low latency, and a highly efficient usage of energy and spectrum resources, in order to support a myriad of connected devices and new services stemming from paradigms like the Internet of Things [1]. Non-orthogonal multiple access (NOMA) has been considered as a key enabling

Copyright (c) 2015 IEEE. Personal use of this material is permitted. However, permission to use this material for any other purposes must be obtained from the IEEE by sending a request to pubs-permissions@ieee.org.

A. Tregancini and E. E. Benitez Olivo are with São Paulo State University (UNESP), Campus of São João da Boa Vista, São João da Boa Vista 13876750, Brazil (e-mail: anderson.tregancini@unesp.br, edgar.olivo@unesp.br).

D. P. Moya Osorio is with the Department of Electrical Engineering, Center of Exact Sciences and Technology, Federal University of São Carlos, São Carlos 13565-905, Brazil (e-mail: dianamoya@ufscar.br).

C. H. M. de Lima and H. Alves are with the Centre for Wireless Communications, University of Oulu, Oulu 90014, Finland (email: carlos.lima@oulu.fi, hirley.alves@oulu.fi).

This work was supported in part by the Coordenação de Aperfeiçoamento de Pessoal de Nível Superior - Brazil (CAPES) - Finance Code 001, the Brazilian National Council for Scientific and Technological Development (CNPq), Grants 421850/2018-3 and 428649/2016-5, the São Paulo Research Foundation (FAPESP), Grant 17/20990-6, and the Academy of Finland 6Genesis Flagship, Grant 318927. technology in $5 \mathrm{G}$ networks due to its potential capability to increase the network capacity by enhancing the utilization of the scarce spectrum resources [2]. In contrast to orthogonal multiple access (OMA) techniques, whereby signals coming from different users are orthogonal to each other, NOMA allows multiple users to share the same radio resources by using code- or power-domain multiplexing [3]. In code-domain NOMA, multiplexing is achieved by using codes similarly to code division multiple access (CDMA) systems. In this case, NOMA utilizes specific spreading sequences per user, which can be sparse or non-orthogonal cross-correlation sequences of low correlation coefficient. Alternatively, in power-domain NOMA, different power levels are allocated to each user based on the experimented channel conditions so as to maximize the system performance. In this case, the downlink information can be broadcast by using superposition coding at the transmitter and successive interference cancellation (SIC) at the receivers in order to cancel multiuser interference $[4,5]$. In this study, we will focus on power-domain NOMA.

Recently, the use of NOMA in cooperative 5G deployment scenarios has received a lot of attention, owing to the inherent gains of such cooperative relaying strategies regarding coverage extension and reliability [9-17]. In cooperative relaying networks, a relay node assists the communication between a source and a destination to improve the transmission range or to improve the reliability by leveraging spatial diversity [6]. According to the relay behavior, two main protocols are widely known, namely: decode-and-forward (DF), whereby the relay decodes and re-encodes the information signal before forwarding it [7]; and amplify-and-forward (AF), whereby the relay merely amplifies the received signal coming from the source and forwards it to the destination $[8]^{1}$. Herein, we focus on the AF protocol, as it requires less computationally demanding processing at the relay, when compared to the other relaying protocols. Lately, a few works have addressed the joint use of NOMA and cooperative communications, whose contributions can be categorized from two perspectives [3]: $(i)$ cooperative NOMA, whereby NOMA users with good channel conditions, referred to as near or strong users, act as relays to assist NOMA users with poor channel conditions, referred to as far or weak users [9]; and (ii) relay-aided NOMA transmission, through which the communication among NOMA users is

\footnotetext{
${ }^{1}$ Other alternative relaying protocols to DF and AF are: compress-andforward $(\mathrm{CF})$, in which the relay does not decode but instead employs Wyner$\mathrm{Ziv}$ coding to compress the received signal before forwarding it, and quantizeand-forward (QF), by which the relay quantizes the received signal and then forwards it to the destination.
} 
assisted by one or more dedicated relays [10-17].

For example, from the perspective of cooperative NOMA schemes, in [9], the outage probability and ergodic sum rate were derived for a cooperative NOMA system, considering arbitrary and optimal power allocation schemes with fullduplex (FD) users operating as DF relays.

From the perspective of relay-aided NOMA transmission schemes, the authors in [10] and [11] proposed a coordinated transmission strategy for deployment scenarios with a base station having a direct link to one user, while simultaneously communicating with another user through a relay, wherein NOMA was used to enable the receivers to acquire other user's information for interference cancellation. For those systems, analytical expressions for the outage probability and the ergodic sum capacity were obtained. In particular, in [10] the relay was assumed to operate in half-duplex (HD) mode, whereas in [11] the FD relaying mode was considered. In [12], a contract-based incentive mechanism along with a relay selection scheme was proposed for a multi-channel dual-hop cooperative NOMA system, which is composed by one base station, multiple users, and multiple relays that can potentially assist the NOMA transmission in HD relaying mode. In [13], the outage probability and the ergodic sum rate of a NOMAbased cooperative network over Nakagami- $m$ channels were derived, where the source was assumed to simultaneously communicate with multiple destinations through an HD-AF relay. In [14], the performance in terms of the outage probability and sum rate for a NOMA scheme in a cooperative network with multiple HD-AF relays were studied, considering partial relay selection (PRS). In [15], the outage probability for an optimal user-relay pair selection scheme, which maximizes the quality of service in a NOMA scenario, was investigated. In [16], the performance in terms of the outage probability for different relay selection schemes, considering a NOMAbased cooperative network scenario with multiple HD-DF relays, was analyzed. In [17], the outage performance and the ergodic sum rate were investigated for a NOMA-based relaying network over Nakagami- $m$ channels with a single FDAF relay; however, therein a fading-free SI link at the relay was assumed. The benefits of relay-aided NOMA systems are also assessed in [18-22] and references thereof. For instance, the outage probability for a NOMA-based relaying system with one transmitter communicating with two receivers via multiple relays is assessed in [18]. Therein, two-stage DF and AF relaying approaches wherein users are ordered based on their quality of service (QoS) requirements are investigated. Besides, in [19], two optimal relay selection schemes for cooperative NOMA with fixed and adaptive power allocations at the relay nodes, which operate under the DF protocol, are introduced. Both works in $[18,19]$ focused on HD-based relaying scenarios. On the other hand, in [20], a virtual FD relaying scheme for cooperative NOMA-based systems was introduced and its performance was analyzed in terms of the outage probability and the respective ergodic rate. Therein, two HDAF relays were employed to emulate the FD relaying operation by using a successive relaying technique. Aiming to balance spectral efficiency and energy conservation, the authors in [21] proposed an optimal power allocation to maximize the energy efficiency of a cooperative NOMA system employing FD twoway cognitive transmission, while maintaining the bit rate requirements of NOMA users. Considering both perfect and imperfect SIC, a FD cooperative NOMA system in which two users intend to exchange information with the assistance of one FD-DF relay was introduced in [22], and its performance was then evaluated through the outage probability and achievable ergodic rate.

Equally important, FD relaying has emerged as a promising technique to provide improved spectral efficiency in wireless networks despite of the inherent self-interference problem between the transmit and receive antennas at the relay transceiver. In this respect, several research efforts have been made by using SI cancellation techniques in the propagation, analog, and digital domains [23,24]. For example, an aggregate SI suppression close to $100 \mathrm{~dB}$ was reported in [24] by combining an advanced antenna design based on resonant wavetraps and digital cancellation. Although FD relaying has been extensively addressed in OMA-based cooperative networks [25-27], a few works have tackled FD in cooperative relaying networks based on NOMA [11,17,21,22]. In fact, many scenarios for the combined use of NOMA and cooperative communications based on FD relaying techniques remain still unexplored.

In this regard, this contribution investigates relay-aided NOMA cooperative network consisting of one source and two destinations $^{2}$, which communicate via one relay chosen from a cluster of FD-AF relays. Bearing in mind a lesser burden in terms of the required feedback overhead, a PRS scheme based on the channel state information (CSI) of the sourcerelay links is employed to select the relay that cooperates in the communication process between the source and destinations. Besides, we consider an imperfect SI cancellation at the FD relay nodes, so that the effect of a residual SI on the system performance is considered. In this scenario, the stronger destination undergoing the best channel condition uses a SIC strategy to firstly decode the information of the weaker destination with worst channel condition. Thereafter, the stronger destination decodes its own information. For the considered setup, the system performance is evaluated in terms of the outage probability and the ergodic capacity for each of the NOMA destination nodes.

The main contributions of this work are summarized as follows:

- Analytical expressions for the end-to-end (E2E) instantaneous received signal-to-interference-plus-noise ratio (SINR) are obtained for each destination.

- Exact single-fold integral expressions for the outage probability of both destinations are derived. These expressions are validated through Monte Carlo simulations.

- Closed-form expressions are obtained from a lowerbound analysis for the outage probability of each destination, which, interestingly, prove excellent approximations

\footnotetext{
${ }^{2} \mathrm{~A}$ large number of destinations multiplexed in the power domain might not be feasible in practice, since the processing complexity of SIC at receivers grows non-linearly with the number of users. This complexity is more evident when SIC error propagation is assumed; therefore, two power-domain NOMA users is a practical assumption [28-31].
} 
to the corresponding exact outage probabilities in the medium-to-high signal-to-noise ratio (SNR) regime.

- Approximate closed-form expressions are derived by using the Gauss-Laguerre method, which are shown to be highly accurate over the entire range of SNR values.

- An asymptotic analysis of the outage probability at highSNR is carried out from which closed-form expressions are obtained, corroborating the zero-diversity problem inherent to FD relaying-based systems in this SNR regime.

- Approximate closed-form expressions for the ergodic capacity of the NOMA users are obtained for the proposed scheme, which prove very tight to the actual performance in the medium-to-high SNR regime.

- The impact of key system parameters on the outage performance was evaluated from the obtained analytical expressions, including the number of relays, the power allocation factor between NOMA destinations, and the level of residual SI.

- A performance comparison between the proposed scheme and the HD relay-aided NOMA system counterpart is also provided. In addition, the impact of number of relays on the sum rate performance of the proposed NOMA scheme is examined.

The remainder of the paper is organized as follows. The system and signal models are introduced in Section II. The exact, approximate, lower-bound, and asymptotic analyses of the outage probability are performed in Section III. Section IV provides analytical expressions for the ergodic capacity of both NOMA users, as well as for the system sum rate, derived from exact and approximate analyses. Numerical results are illustrated and discussed in Section V. Finally, the conclusions of this work are drawn in Section VI.

Notation: Throughout this paper, $f_{Z}(\cdot)$ and $F_{Z}(\cdot)$ denote the probability density function (PDF) and the cumulative distribution function (CDF) of a random variable (RV) $Z$, respectively, $\mathcal{C N}\left(\mu, \sigma^{2}\right)$ denotes a complex circularly-symmetric Gaussian distribution with mean $\mu$ and variance $\sigma^{2}, \mathrm{E}[\cdot]$ denotes expectation, and $\operatorname{Pr}(\cdot)$ denotes probability. In addition, $\mathrm{Ei}(\cdot)$ denotes the exponential integral function [33, eq. (5.1.2)].

\section{System And Signal Models}

Consider a dual-hop relaying network as depicted in Fig. 1, in which a source $\mathrm{S}$ intends to communicate with two NOMA users, $\left\{\mathrm{D}_{\ell}\right\}_{\ell=1}^{2}$, using the assistance of one out of $K$ variablegain AF relays, $\left\{\mathrm{R}_{k}\right\}_{k=1}^{K}$, which operate in FD mode. The nodes $\mathrm{S}, \mathrm{D}_{1}$, and $\mathrm{D}_{2}$ are single-antenna devices, whereas the relays are equipped with two antennas enabling FD relaying operation. However, such relaying mode is degraded by strong SI, which cannot be perfectly canceled, thus the relays are assumed to suffer from residual SI. The relay that participates of the communication process is selected by considering a PRS scheme based on CSI of the first-hop $\left(S \rightarrow R_{k}\right)$ links. All nodes are considered to operate on a time-division multiple access (TDMA) basis. In addition, all links are assumed to undergo independent block Rayleigh fading ${ }^{3}$ and additive

\footnotetext{
${ }^{3}$ All channel coefficients are assumed to remain constant during each fading block, while changing independently over consecutive blocks.
}

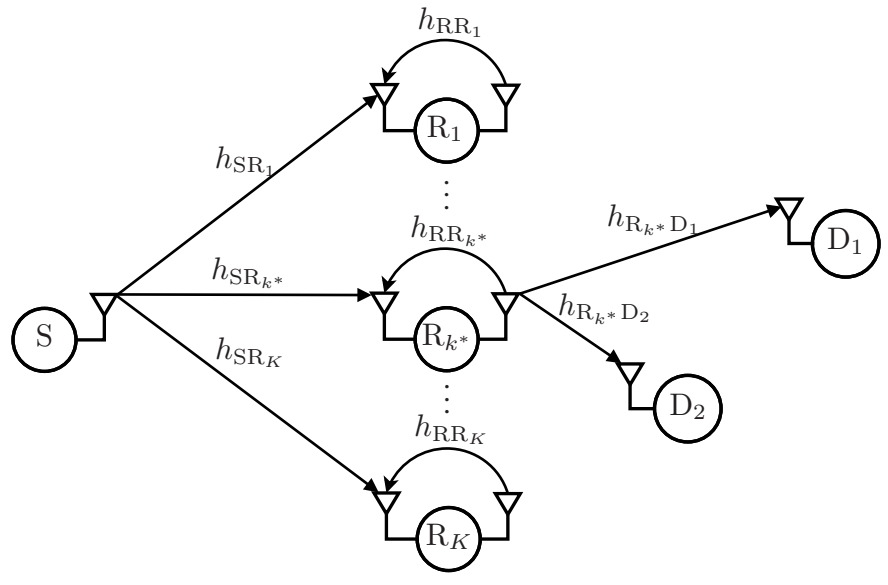

Fig. 1. Full-duplex relay-aided NOMA network deployment model, in which a source $(\mathrm{S})$ communicates simultaneously in the power domain with two destinations $\left(\mathrm{D}_{1}\right.$ and $\left.\mathrm{D}_{2}\right)$, under the assistance of one out of $K$ FD-AF relays $\left(\mathrm{R}_{k}\right)$, using partial relay selection. All the links are subject to fading and noise. Note that the relays suffer from self-interference.

white Gaussian noise (AWGN). Hence, the instantaneous received SNRs at the first-hop and second-hop $\left(\mathrm{R}_{k} \rightarrow \mathrm{D}_{\ell}\right)$ links can be given, respectively, as $X_{k}=\left|h_{\mathrm{SR}_{k}}\right|^{2} P_{\mathrm{S}} / N_{0}$ and $Y_{k \ell}=\left|h_{\mathrm{R}_{k} \mathrm{D}_{\ell}}\right|^{2} P_{\mathrm{R}} / N_{0}$, where $h_{\mathrm{SR}_{k}} \sim \mathcal{C N}\left(0, \Omega_{\mathrm{SR}_{k}}\right)$ and $h_{\mathrm{R}_{k} \mathrm{D}_{\ell}} \sim \mathcal{C N}\left(0, \Omega_{\mathrm{R}_{k} \mathrm{D}_{\ell}}\right)$ are the corresponding channel coefficients, with $\Omega_{\mathrm{SR}_{k}}=\mathrm{E}\left[\left|h_{\mathrm{SR}_{k}}\right|^{2}\right]$ and $\Omega_{\mathrm{R}_{k} \mathrm{D}_{\ell}}=\mathrm{E}\left[\left|h_{\mathrm{R}_{k} \mathrm{D}_{\ell}}\right|^{2}\right]$ being the average channel gains; $P_{\mathrm{S}}$ and $P_{\mathrm{R}}$ are the transmit powers at $\mathrm{S}$ and $\mathrm{R}_{k}$; and $N_{0}$ is the mean noise power at the receivers. Also, the residual SI links at the relays, after some stages of imperfect SI cancellation, are assumed to be subject to independent and identically distributed (i.i.d.) Rayleigh fading [26], such that the instantaneous received SNR at the SI link of the $k$ th relay is given as $U_{k}=\left|h_{\mathrm{RR}_{k}}\right|^{2} P_{\mathrm{R}} / N_{0}$, where $h_{\mathrm{RR}_{k}} \sim \mathcal{C N}\left(0, \Omega_{\mathrm{RR}_{k}}\right)$ is the corresponding channel coefficient, with $\Omega_{\mathrm{RR}_{k}}=\mathrm{E}\left[\left|h_{\mathrm{RR}_{k}}\right|^{2}\right]$ being the average channel gain. In the proposed setup, $\mathrm{D}_{2}$ is assumed to undergo better channel conditions than $\mathrm{D}_{1}$, so that $\left|h_{\mathrm{R}_{k} \mathrm{D}_{1}}\right|^{2}<\left|h_{\mathrm{R}_{k} \mathrm{D}_{2}}\right|^{2}$.

Herein we focus on a coverage extension scenario, in which due to physical obstacles or severe radio channel degradation, the direct link between $\mathrm{S}$ and $\mathrm{D}_{\ell}$ is unavailable. Thus, the communication process is feasible only through the cooperative relays. By considering a power-domain NOMA scheme, $S$ first broadcasts the information messages intended to each destination simultaneously, such that the transmitted signal at time $t$ is given by

$$
s(t)=\sqrt{a_{1} P_{\mathrm{S}}} x_{1}(t)+\sqrt{a_{2} P_{\mathrm{S}}} x_{2}(t),
$$

where $x_{1}(t)$ and $x_{2}(t)$ are the information signals for users $\mathrm{D}_{1}$ and $\mathrm{D}_{2}$, respectively; and $a_{1}$ and $a_{2}$ are the power allocation factors assigned to $\mathrm{D}_{1}$ and $\mathrm{D}_{2}$ according to the NOMA scheme, such that the relationships $a_{1}>a_{2}$ and $a_{1}+a_{2}=1$ must be satisfied $[3,14]$. Then, the received signal at the $k$ th relay is given by

$$
r_{k}(t)=h_{\mathrm{SR}_{k}} \sqrt{P_{\mathrm{S}}} s(t)+h_{\mathrm{RR}_{k}} \sqrt{P_{\mathrm{R}}} v_{k}(t)+n_{\mathrm{R}_{k}}(t),
$$


where

$$
v_{k}(t)=\beta r_{k}\left(t-T_{d}\right)
$$

is the signal retransmitted by the $k$ th $\mathrm{FD}$ relay at time $t$, with $\beta$ being the amplification factor relative to the $\mathrm{AF}$ relaying protocol and $T_{d}$ being the processing delay ${ }^{4}$, and $n_{\mathrm{R}_{k}}(t) \sim \mathcal{C} \mathcal{N}\left(0, N_{0}\right)$ is the AWGN component at $\mathrm{R}_{k}$. Therefore, after performing recursive substitutions of (2) into (3), the signal retransmitted by the $k$ th relay can be given as

$$
\begin{aligned}
v_{k}(t)= & \beta \sum_{j=1}^{L}\left(\beta h_{\mathrm{RR}_{k}} \sqrt{P_{\mathrm{R}}}\right)^{j-1}\left\{h _ { \mathrm { SR } _ { k } } \left[\sqrt{a_{1} P_{\mathrm{S}}} x_{1}\left(t-j T_{d}\right)\right.\right. \\
& \left.\left.+\sqrt{a_{2} P_{\mathrm{S}}} x_{2}\left(t-j T_{d}\right)\right]+n_{\mathrm{R}_{k}}\left(t-j T_{d}\right)\right\}
\end{aligned}
$$

where $L$ is the block length that typically contains a very large number of symbols, so that $T_{d} \ll T_{L}$, where $T_{L}=L T_{s}$ is the block duration, with $T_{s}$ being the symbol period.

To better understand the nature of the expression in (4), we can rewrite it as follows

$$
\begin{aligned}
v_{k}(t) & =\underbrace{\beta h_{\mathrm{SR}_{k}} \sqrt{a_{1} P_{\mathrm{S}}} x_{1}\left(t-T_{d}\right)}_{\text {desired signal of } \mathrm{D}_{1}}+\underbrace{\beta h_{\mathrm{SR}_{k}} \sqrt{a_{2} P_{\mathrm{S}}} x_{2}\left(t-T_{d}\right)}_{\text {desired signal of } \mathrm{D}_{2}} \\
& +\underbrace{\beta n_{\mathrm{R}_{k}}\left(t-T_{d}\right)}_{\text {noise signal }} \\
& +\beta \sum_{j=2}^{L}\left(\beta h_{\mathrm{RR}_{k}} \sqrt{P_{\mathrm{R}}}\right)^{j-1}\left\{h _ { \mathrm { SR } _ { k } } \left[\sqrt{a_{1} P_{\mathrm{S}}} x_{1}\left(t-j T_{d}\right)\right.\right. \\
& +\underbrace{\left.\left.\sqrt{a_{2} P_{\mathrm{S}}} x_{2}\left(t-j T_{d}\right)\right]+n_{\mathrm{R}_{k}}\left(t-j T_{d}\right)\right\},}_{\text {self-interference signal }}
\end{aligned}
$$

from which the following four components can be distinguished: ( $i$ ) desired signal of $\mathrm{D}_{1}$, (ii) desired signal of $\mathrm{D}_{2}$, (iii) noise signal, and (iv) SI signal. Now, without loss of generality, by assuming that $\mathrm{E}\left[\left|x_{1}(t)\right|^{2}\right]=\mathrm{E}\left[\left|x_{2}(t)\right|^{2}\right]=1$, the mean power of the signal retransmitted by the $k$ th relay can be obtained from (5) as

$$
\begin{aligned}
\mathrm{E} & {\left[\left|v_{k}(t)\right|^{2}\right] } \\
& =\beta^{2} \sum_{j=1}^{L}\left(\beta^{2}\left|h_{\mathrm{RR}_{k}}\right|^{2} P_{\mathrm{R}}\right)^{j-1}\left[\left|h_{\mathrm{SR}_{k}}\right|^{2} P_{\mathrm{S}}+N_{0}\right] \\
& \stackrel{(a)}{=} \beta^{2}\left(1-\beta^{2}\left|h_{\mathrm{RR}_{k}}\right|^{2} P_{\mathrm{R}}\right)^{-1}\left[\left|h_{\mathrm{SR}_{k}}\right|^{2} P_{\mathrm{S}}+N_{0}\right],
\end{aligned}
$$

where step $(a)$ follows by considering a convergent geometric sequence, with $\beta^{2}\left|h_{\mathrm{RR}_{k}}\right|^{2} P_{\mathrm{R}}<1$. Thus, the amplification factor at the AF relays can be obtained as

$$
\beta^{2}=\left[\left|h_{\mathrm{SR}_{k}}\right|^{2} P_{\mathrm{S}}+\left|h_{\mathrm{RR}_{k}}\right|^{2} P_{\mathrm{R}}+N_{0}\right]^{-1} .
$$

On the other hand, the receive signals at $D_{1}$ and $D_{2}$ from $R_{k}$ are given, respectively, by

$$
y_{\mathrm{D}_{1}}(t)=h_{\mathrm{R}_{k} \mathrm{D}_{1}} \sqrt{P_{\mathrm{R}}} v_{k}(t)+n_{\mathrm{D}_{1}}(t),
$$

${ }^{4}$ Herein, the processing delay $T_{d}$ is assumed to be greater than the symbol period, in order to guarantee decorrelation between the simultaneously received and transmitted symbols by the relay at a given time instant [25].

$$
y_{\mathrm{D}_{2}}(t)=h_{\mathrm{R}_{k} \mathrm{D}_{2}} \sqrt{P_{\mathrm{R}}} v_{k}(t)+n_{\mathrm{D}_{2}}(t),
$$

where $n_{\mathrm{D}_{\ell}}(t)$, with $\ell=1,2$, are the AWGN components at each NOMA destination.

From the above signal analysis, after some mathematical manipulations, the instantaneous received E2E SINR at $D_{1}$ can be obtained as

$$
\gamma_{\mathrm{D}_{1}}=\frac{a_{1} X_{k} Y_{k 1}}{a_{2} X_{k} Y_{k 1}+Y_{k 1} U_{k}+X_{k}+Y_{k 1}+U_{k}+1} .
$$

Moreover, according to NOMA, the strong user $\mathrm{D}_{2}$ must first decode the message intended to the weak user $D_{1}$. This is feasible since $a_{1}>a_{2}$. Then, once weak user's message is obtained, $\mathrm{D}_{2}$ is able to decode its own message by performing a SIC technique. Therefore, the instantaneous received E2E SINRs at $\mathrm{D}_{2}$, relative to the weak user's information and to its own information can be expressed, respectively, as

$$
\begin{aligned}
\gamma_{\mathrm{D}_{12}} & =\frac{a_{1} X_{k} Y_{k 2}}{a_{2} X_{k} Y_{k 2}+Y_{k 2} U_{k}+X_{k}+Y_{k 2}+U_{k}+1}, \\
\gamma_{\mathrm{D}_{2}} & =\frac{a_{2} X_{k} Y_{k 2}}{Y_{k 2} U_{k}+X_{k}+Y_{k 2}+U_{k}+1} .
\end{aligned}
$$

On the other hand, by considering a PRS criterion based on the first-hop CSI, the index of the selected relay which assists in the communication process between the source and NOMA destinations is given by

$$
k^{*}=\arg \max _{k}\left(Z_{k}\right),
$$

where $Z_{k}$ is the first-hop SINR at the $k$ th relay, given as

$$
Z_{k} \triangleq \frac{X_{k}}{U_{k}+1} \text {. }
$$

\section{Outage Probability Analysis}

\section{A. Exact Analysis}

In this section, the exact outage probability for each NOMA destination, $\mathrm{D}_{1}$ and $\mathrm{D}_{2}$, is derived. To simplify the analysis, the SNR thresholds relative to the target quality of service of each user, $\tau_{1}$ and $\tau_{2}$, which define the outage events, are assumed to be equal, that is, $\tau_{1}=\tau_{2}=\tau$. In addition, it is considered that $P_{\mathrm{S}}=P_{\mathrm{R}}=P$. Hereafter, aiming at a simpler notation, under the assumption of i.i.d. channels, we consider that $\Omega_{\mathrm{SR}_{k}}=\Omega_{\mathrm{SR}}, \Omega_{\mathrm{R}_{k} \mathrm{D}_{\ell}}=\Omega_{\mathrm{RD}_{\ell}}$ and $\Omega_{\mathrm{RR}_{k}}=\Omega_{\mathrm{RR}}$.

1) Outage probability at $\mathrm{D}_{1}$ : An outage event occurs at $\mathrm{D}_{1}$ when its instantaneous received E2E SINR, given as in (10), falls below the threshold $\tau$. Thus, the outage probability at user $\mathrm{D}_{1}$ is given as

$$
\begin{aligned}
& \mathrm{OP}_{1} \\
& \quad=\operatorname{Pr}\left(\gamma_{\mathrm{D}_{1}}<\tau\right) \\
& =\operatorname{Pr}\left(\frac{a_{1} X_{k^{*}} Y_{k^{*}}}{a_{2} X_{k^{*}} Y_{k^{*} 1}+Y_{k^{*}} U_{k^{*}}+X_{k^{*}}+Y_{k^{*}}+U_{k^{*}}+1}<\tau\right),
\end{aligned}
$$

where $k^{*}$ refers to the index of the selected relay, according to the criterion in (13). From (15), an exact analytical expression for the outage probability of $D_{1}$ is obtained, as shown next

Proposition 1. In a relay-aided NOMA system with two destinations, in which PRS among $K$ FD-AF relays is employed, 
the exact outage probability for the weak user, $\mathrm{D}_{1}$, is given by

$$
\begin{aligned}
& \mathrm{OP}_{1}=1-e^{-\frac{\tilde{\tau}}{\mathrm{\gamma D}_{1}}} \\
& +\frac{e^{-\frac{\tilde{\tau}}{\bar{\gamma}_{1}}}}{\bar{\gamma}_{\mathrm{RD}_{1}}} \int_{0}^{\infty}\left[1-\frac{e^{-\frac{\left(y_{1}+\tilde{\tau}+1\right) \tilde{\tau}}{y_{1} \bar{\gamma}_{\mathrm{SR}}}}}{1+\frac{\bar{\gamma}_{\mathrm{RR}}\left(y_{1}+\tilde{\tau}+1\right) \tilde{\tau}}{y_{1} \bar{\gamma}_{\mathrm{SR}}}}\right]^{K} e^{-\frac{y_{1}}{\bar{\gamma}_{\mathrm{RD}}}} \mathrm{d} y_{1},
\end{aligned}
$$

where $\bar{\gamma}_{\mathrm{SR}}=\gamma_{0} \Omega_{\mathrm{SR}}, \bar{\gamma}_{\mathrm{RD}_{1}}=\gamma_{0} \Omega_{\mathrm{RD}_{1}}$, and $\bar{\gamma}_{\mathrm{RR}}=\gamma_{0} \Omega_{\mathrm{RR}}$ denote the average received SNRs at the $\mathrm{S} \rightarrow \mathrm{R}_{k}, \mathrm{R}_{k} \rightarrow \mathrm{D}_{1}$, and SI links, respectively, with $\gamma_{0}=P / N_{0}$ being the transmit $\mathrm{SNR}$, and $\widetilde{\tau} \triangleq \tau /\left(a_{1}-a_{2} \tau\right)$.

Proof. See Appendix A.

2) Outage probability at $\mathrm{D}_{2}$ : The strong user $\mathrm{D}_{2}$ is considered to be in outage when one of the following events occur: (i) the instantaneous received E2E SINR at $\mathrm{D}_{2}$ relative to weak user's information, $\gamma_{\mathrm{D}_{12}}$, is below the threshold $\tau$, thus implying that the SIC process performed at $\mathrm{D}_{2}$ to remove $\mathrm{D}_{1}$ 's information fails, or ( ii) after successfully executing the SIC process, the instantaneous received E2E SINR at $\mathrm{D}_{2}$ relative to its own information, $\gamma_{\mathrm{D}_{2}}$, is below $\tau$. Therefore, the outage probability for $\mathrm{D}_{2}$ can be formulated as

$$
\mathrm{OP}_{2}=\operatorname{Pr}\left(\gamma_{\mathrm{D}_{12}}<\tau\right)+\operatorname{Pr}\left(\gamma_{\mathrm{D}_{12}}>\tau, \gamma_{\mathrm{D}_{2}}<\tau\right) .
$$

Then, by substituting (11) and (12) into (17), an exact analytical expression for the outage probability of $\mathrm{D}_{2}$ is obtained, as presented next.

Proposition 2. In a relay-aided NOMA system with two destinations, in which PRS among $K$ FD-AF relays is employed, the exact outage probability for the strong user, $\mathrm{D}_{2}$, is given by

$$
\begin{aligned}
& \mathrm{OP}_{2}=1-e^{-\frac{\theta}{\bar{\gamma}_{\mathrm{RD}}}} \\
& +\frac{e^{-\frac{\theta}{\bar{\gamma}_{\mathrm{RD}_{2}}}}}{\bar{\gamma}_{\mathrm{RD}_{2}}} \int_{0}^{\infty}\left[1-\frac{e^{-\frac{\left(y_{2}+\theta+1\right) \theta}{y_{2} \bar{\gamma}_{\mathrm{SR}}}}}{1+\frac{\bar{\gamma}_{\mathrm{RR}}\left(y_{2}+\theta+1\right) \theta}{y_{2} \bar{\gamma}_{\mathrm{SR}}}}\right]^{K} e^{-\frac{y_{2}}{\bar{\gamma}_{\mathrm{RD}_{2}}} \mathrm{~d} y_{2},}
\end{aligned}
$$

where $\bar{\gamma}_{\mathrm{RD}_{2}}=\gamma_{0} \Omega_{\mathrm{RD}_{2}}$ is the average received SNR at the $\mathrm{R}_{k} \rightarrow \mathrm{D}_{2}$ link, and $\theta \triangleq \max \left\{\widetilde{\tau}, \tau^{\prime}\right\}$, with $\tau^{\prime} \triangleq \tau / a_{2}$.

Proof. See Appendix B.

Although the outage probability expressions given in (16) and (18) can be readily evaluated through standard computing software, it is desirable to attain closed-form expressions for those outage probabilities in order to reduce even more the computational complexity. For this purpose, in the next subsections, we introduce simple and useful closed-form expressions derived from lower bound-, approximation-, and asymptotebased analyses. Importantly, as will be seen in Section V, these new analytical expressions prove to be very tight to exact outage performance along the entire SNR range.

\section{B. Lower-Bound Analysis}

From the well-known upper bound for the harmonic mean of two arbitrary SNR values $A$ and $B$, given by $A B /(A+B)<$ $\min \{A, B\}$ [27], lower-bound expressions for the outage probability of each NOMA destination, $\mathrm{D}_{1}$ and $\mathrm{D}_{2}$, can be derived in closed form. Such lower-bound expressions result in excellent approximations to the exact outage probabilities given in (16) and (18), at high SNR, and are introduced in the following propositions.

Proposition 3. In a relay-aided NOMA system with two destinations, in which PRS among $K$ FD-AF relays is employed, a lower bound for the outage probability of the weak user, $D_{1}$, is given in closed form as

$$
\mathrm{OP}_{1}^{\mathrm{LB}}=1-\left(1-\left[1-\frac{e^{-\frac{\tilde{\tau}}{\bar{\gamma}_{\mathrm{SR}}}}}{1+\frac{\bar{\gamma}_{\mathrm{RR}}}{\bar{\gamma}_{\mathrm{SR}}}}\right]^{K}\right) e^{-\frac{\tilde{\tau}}{\overline{\mathrm{RD}}_{1}}} .
$$

Proof. From (10), by using the definition of $Z_{k}$ in (14), it follows that

$$
\begin{aligned}
\mathrm{OP}_{1} & \stackrel{(f)}{>} \operatorname{Pr}\left(\frac{a_{1} Z_{k^{*}} Y_{k^{*} 1}}{a_{2} Z_{k^{*}} Y_{k^{*} 1}+Z_{k^{*}}+Y_{k^{*} 1}}<\tau\right) \\
& =\operatorname{Pr}\left(\frac{Z_{k^{*}} Y_{k^{*} 1}}{Z_{k^{*}}+Y_{k^{*} 1}}<\widetilde{\tau}\right) \\
& >\operatorname{Pr}\left(\min \left\{Z_{k^{*}}, Y_{k^{*} 1}\right\}<\widetilde{\tau}\right) \\
& \stackrel{(g)}{=} 1-\operatorname{Pr}\left(Z_{k^{*}}>\widetilde{\tau}, Y_{k^{*} 1}>\widetilde{\tau}\right) \\
& =1-\left(1-\left(F_{Z}(\widetilde{\tau})\right)^{K}\right)\left(1-F_{Y_{1}}(\widetilde{\tau})\right)
\end{aligned}
$$

where step $(f)$ follows from considering a high SNR regime, and step $(g)$ follows from considering the identity $\operatorname{Pr}(\min \{A, B\})=1-\operatorname{Pr}\{\bar{A}, \bar{B}\} \quad$ [34]. Thus, after the appropriate substitutions in (20), a lower bound for the outage probability of $\mathrm{D}_{1}$ is obtained as in (19).

Proposition 4. In a relay-aided NOMA system with two destinations, in which PRS among $K$ FD-AF relays is employed, a lower bound for the outage probability of the strong user, $\mathrm{D}_{2}$, is given in closed form as

$$
\mathrm{OP}_{2}^{\mathrm{LB}}=1-\left(1-\left[1-\frac{e^{-\frac{\theta}{\bar{\gamma}_{\mathrm{SR}}}}}{1+\frac{\bar{\gamma}_{\mathrm{RR}} \theta}{\bar{\gamma}_{\mathrm{SR}}}}\right]^{K}\right) e^{-\frac{\theta}{\bar{\gamma}_{\mathrm{RD}}}} .
$$

Proof. By following a similar procedure to that presented in the proof of Proposition 3, it follows from (12) that

$$
\begin{aligned}
\mathrm{OP}_{2} & >\operatorname{Pr}\left(\frac{Z_{k^{*}} Y_{k^{*}}}{Z_{k^{*}}+Y_{k^{*} 2}}<\theta\right) \\
& >\operatorname{Pr}\left(\min \left\{Z_{k^{*}}, Y_{k^{*} 2}\right\}<\theta\right) \\
& =1-\operatorname{Pr}\left(Z_{k^{*}}>\theta, Y_{k^{*} 2}>\theta\right) \\
& =1-\left(1-\left(F_{Z}(\theta)\right)^{K}\right)\left(1-F_{Y_{2}}(\theta)\right),
\end{aligned}
$$

from which, after the appropriate substitutions, a lower bound for the outage probability of $\mathrm{D}_{2}$ is obtained as in (21).

\section{Approximate Analysis}

As an alternative to the previous approach, an approximate analysis for the outage probability of each NOMA destination is proposed to attain simpler expressions for the performance evaluation of the system under investigation. In particular, approximate closed-form expressions for the exact outage 
probabilities of users $\mathrm{D}_{1}$ and $\mathrm{D}_{2}$, given in integral form as in (16) and (18) are obtained in the following propositions by applying the Gauss-Laguerre quadrature method [33, eq. (25.5.45)]. According to this method, we have that

$$
\int_{0}^{\infty} f(x) e^{-x} d x=\sum_{i=1}^{n} \omega_{i} f\left(x_{i}\right)+E_{n},
$$

that is, integrals given as in (23) can be approximated by a weighted sum of integrand samples, where $x_{i}$ is the $i$ th root of the $n$ th-order Laguerre polynomial $L_{n}(x)$, $\omega_{i}=x_{i} /\left[(n+1)\left(L_{n+1}\left(x_{i}\right)\right)\right]^{2}$ is the $i$ th weight and $E_{n}=$ $(n !)^{2} f^{(2 n)}(\xi) /(2 n)$ ! is the truncate error, with $0<\xi<\infty$.

Proposition 5. In a relay-aided NOMA system with two destinations, in which PRS among $K$ FD-AF relays is employed, an approximate closed-form expression for the outage probability of the weak user, $\mathrm{D}_{1}$, is given by

$$
\mathrm{OP}_{1} \approx 1-e^{-\frac{\tilde{\tau}}{\mathrm{R}_{1}}}+e^{-\frac{\tilde{\tau}}{\mathrm{R}_{1}}} \sum_{i=1}^{n} \omega_{i} f_{1}\left(x_{i}\right),
$$

where

$$
f_{1}(x)=\left[1-\frac{e^{-\frac{\left(x \bar{\gamma}_{\left.\mathrm{RD}_{1}+\tilde{\tau}+1\right) \tilde{\tau}}\right.}{x \bar{\gamma}_{\mathrm{RD}} \bar{\gamma}_{\mathrm{SR}}}}}{1+\frac{\bar{\gamma}_{\mathrm{RR}}\left(x \bar{\gamma}_{\left.\mathrm{RD}_{1}+\tilde{\tau}+1\right) \widetilde{\tau}}\right.}{x \bar{\gamma}_{\mathrm{RD}} \bar{\gamma}_{\mathrm{SR}}}}\right]^{K} .
$$

Proof. The approximate expression in (24) follows in a straightforward manner from applying (23) to the integral in (16).

Proposition 6. In a relay-aided NOMA system with two destinations, in which PRS among $K$ FD-AF relays is employed, an approximate closed-form expression for the outage probability of the strong user, $\mathrm{D}_{2}$, is given by

$$
\mathrm{OP}_{2} \approx 1-e^{-\frac{\theta}{\overline{\mathrm{RD}_{2}}}}+e^{-\frac{\theta}{\overline{\mathrm{RD}_{2}}}} \sum_{i=1}^{n} \omega_{i} f_{2}\left(x_{i}\right),
$$

where

$$
f_{2}(x)=\left[1-\frac{e^{-\frac{\left(x \bar{\gamma}_{\mathrm{RD}_{2}}+\theta+1\right) \theta}{x \bar{\gamma}_{\mathrm{RD}} \bar{\gamma}_{\mathrm{SR}}}}}{1+\frac{\bar{\gamma}_{\mathrm{RR}}\left(x \bar{\gamma}_{\mathrm{RD}_{2}}+\theta+1\right) \theta}{x \bar{\gamma}_{\mathrm{RD}_{2}} \bar{\gamma}_{\mathrm{SR}}}}\right]^{K} .
$$

Proof. Similarly to the latter proposition, the proof follows from applying (23) to the integral in (18).

Remark 1. It is noteworthy that the approximations in (24) and (26) proved to be highly accurate for the entire range of SNR values, even for a reduced number of sum terms (i.e., $n=2$ ), as will be shown in Section V.

\section{Asymptotic Analysis}

By taking into account that the lower-bound expressions obtained in Section III-B render excellent approximations to the exact outage probabilities of the NOMA destinations at high SNR, asymptotic analytical expressions for the outage probability of $D_{1}$ and $D_{2}$ can be derived from (19) and (21), respectively, as follows.
1) Asymptotic Outage probability for $\mathrm{D}_{1}$ : From (19), by using the Maclaurin series for the exponential function and neglecting the higher order terms with respect to the transmit SNR $\gamma_{0}$, an asymptotic closed-form expression for the outage probability of the weak user, $\mathrm{D}_{1}$, at high SNR regime (i.e., as $\left.\gamma_{0} \rightarrow \infty\right)$, is given as

$$
\mathrm{OP}_{1} \simeq\left[\frac{\Omega_{\mathrm{RR}} \widetilde{\tau}}{\Omega_{\mathrm{RR}} \widetilde{\tau}+\Omega_{\mathrm{SR}}}\right]^{K}
$$

2) Asymptotic Outage probability for $\mathrm{D}_{2}$ : Similarly, from (21), an asymptotic closed-form expression for the outage probability of the strong user, $\mathrm{D}_{2}$, at the high $\mathrm{SNR}$ regime is given as

$$
\mathrm{OP}_{2} \simeq\left[\frac{\Omega_{\mathrm{RR}} \theta}{\Omega_{\mathrm{RR}} \theta+\Omega_{\mathrm{SR}}}\right]^{K} .
$$

Remark 2. From (28) and (29), we can conclude that the diversity order of the system under investigation equals zero, as both asymptotic expressions are independent of $\gamma_{0}$. Therefore, at high SNR, the system performance will show an outage floor.

\section{ERGODIC CAPACITY AND SUM RATE ANALYSiS}

In this section, the ergodic capacity ${ }^{5}$ for the NOMA users $\mathrm{D}_{1}$ and $\mathrm{D}_{2}$ and the system sum rate are derived. The sum rate of the considered system is given by $\bar{C}_{\text {sum }}=\bar{C}_{1}+\bar{C}_{2}$, where $\bar{C}_{1}$ and $\bar{C}_{2}$ are the ergodic capacities of the users $\mathrm{D}_{1}$ and $\mathrm{D}_{2}$, respectively.

\section{A. Ergodic capacity at $\mathrm{D}_{1}$}

The instantaneous capacity at weak user $\mathrm{D}_{1}$ is given as

$$
C_{1}=\log _{2}\left(1+\gamma_{D_{1}}\right),
$$

where $\gamma_{D_{1}}$ is the instantaneous received E2E SINR, given as in (10). Therefore, the exact ergodic capacity at $\mathrm{D}_{1}$, defined as the expectation of the expression in (30), can be written as

$$
\begin{aligned}
\bar{C}_{1} & =\mathrm{E}\left[\log _{2}\left(1+\gamma_{\mathrm{D}_{1}}\right)\right] \\
& =\frac{1}{\ln 2} \int_{0}^{\infty} \frac{1-\operatorname{Pr}\left(\gamma_{\mathrm{D}_{1}}<x\right)}{1+x} \mathrm{~d} x,
\end{aligned}
$$

where $\operatorname{Pr}\left(\gamma_{D_{1}}<x\right)$ in (31) is given by (16). From (31), an approximate closed-form expression for the ergodic capacity of $\mathrm{D}_{1}$ can be obtained, as presented in the following proposition.

Proposition 7. In a relay-aided NOMA system with two destinations, in which PRS among $K$ FD-AF relays is employed, an approximated closed-form expression for the ergodic capacity of the weak user, $D_{1}$, is given as in (32), shown at the top of the next page, where

$$
\xi_{1}=\frac{\left(\bar{\gamma}_{\mathrm{SR}}-\bar{\gamma}_{\mathrm{RR}}\right)\left(k \bar{\gamma}_{\mathrm{RD}_{1}}+\bar{\gamma}_{\mathrm{SR}}\right)}{\left(\bar{\gamma}_{\mathrm{SR}} \bar{\gamma}_{\mathrm{RR}} \bar{\gamma}_{\mathrm{RD}_{1}}\right)}
$$

and

$$
\xi_{2}=\frac{\left(a_{2} \bar{\gamma}_{\mathrm{SR}}-\bar{\gamma}_{\mathrm{RR}}\right)\left(k \bar{\gamma}_{\mathrm{RD}_{1}}+\bar{\gamma}_{\mathrm{SR}}\right)}{\left(a_{2} \bar{\gamma}_{\mathrm{SR}} \bar{\gamma}_{\mathrm{RR}} \bar{\gamma}_{\mathrm{RD}_{1}}\right)} .
$$

${ }^{5}$ The well-known ergodic capacity (or Shannon capacity) corresponds to the maximum error-free achievable data rate averaged over all states of a time-varying channel. 


$$
\begin{aligned}
& \bar{C}_{1} \approx \frac{1}{\ln 2} e^{\frac{1}{a_{2} \bar{\gamma}_{\mathrm{RD}}}}\left(\operatorname{Ei}\left(-\frac{1}{a_{2} \bar{\gamma}_{\mathrm{RD}_{1}}}\right)-e^{-\frac{a_{1}}{a_{2} \bar{\gamma}_{\mathrm{RD}}}} \operatorname{Ei}\left(-\frac{1}{\bar{\gamma}_{\mathrm{RD}_{1}}}\right)\right)-\frac{1}{\ln 2} \sum_{k=0}^{K}\left(\begin{array}{c}
K \\
k
\end{array}\right)(-1)^{k} e^{\frac{\bar{\gamma}_{\mathrm{RD}} k+\bar{\gamma}_{\mathrm{SR}}}{a_{2} \mathrm{\gamma D}_{1} \overline{\mathrm{S}}_{\mathrm{SR}}}}\left\{\left(\frac{\bar{\gamma}_{\mathrm{SR}}}{\bar{\gamma}_{\mathrm{SR}}-\bar{\gamma}_{\mathrm{RR}}}\right)^{k}\right. \\
& \times e^{-\frac{a_{1}\left(\bar{\gamma}_{\mathrm{RD}} k+\bar{\gamma}_{\mathrm{SR}}\right)}{a_{2} \bar{\gamma}_{\mathrm{RD}} \bar{\gamma}_{\mathrm{SR}}}}\left[-\mathrm{Ei}\left(-\frac{\bar{\gamma}_{\mathrm{RD}} k+\bar{\gamma}_{\mathrm{SR}}}{\bar{\gamma}_{\mathrm{RR}} \bar{\gamma}_{\mathrm{RD}_{1}}}\right)-\sum_{i=0}^{k-1} \frac{e^{\xi_{1}}}{\xi_{1}^{-i}} \frac{(-1)^{i+1}}{i !}\left(\mathrm{Ei}\left(-\frac{\bar{\gamma}_{\mathrm{RD}_{1}} k+\bar{\gamma}_{\mathrm{SR}}}{\bar{\gamma}_{\mathrm{RR}} \bar{\gamma}_{\mathrm{RD}_{1}}}\right)+e^{-\frac{\bar{\gamma}_{\mathrm{RD}} k+\bar{\gamma}_{\mathrm{SR}}}{\bar{\gamma}_{\mathrm{RR}} \bar{\gamma}_{\mathrm{RD}}}}\right.\right. \\
& \left.\left.\times \sum_{j=1}^{i}\left(\frac{\bar{\gamma}_{\mathrm{RR}} \bar{\gamma}_{\mathrm{RD}}}{\bar{\gamma}_{\mathrm{RD}_{1}} k+\bar{\gamma}_{\mathrm{SR}}}\right)^{j}(-1)^{j-1}(j-1) !\right)\right]-\left(\frac{a_{2} \bar{\gamma}_{\mathrm{SR}}}{a_{2} \bar{\gamma}_{\mathrm{SR}}-\bar{\gamma}_{\mathrm{RR}}}\right)^{k}\left[-\operatorname{Ei}\left(-\frac{\bar{\gamma}_{\mathrm{RD}} k+\bar{\gamma}_{\mathrm{SR}}}{a_{2} \bar{\gamma}_{\mathrm{SR}} \bar{\gamma}_{\mathrm{RD}_{1}}}\right)-\sum_{i=0}^{k-1} \frac{e^{\xi_{2}}}{\xi_{2}^{-i}} \frac{(-1)^{i+1}}{i !}\right. \\
& \left.\left.\times\left(\operatorname{Ei}\left(-\frac{\bar{\gamma}_{\mathrm{RD}} k+\bar{\gamma}_{\mathrm{SR}}}{\bar{\gamma}_{\mathrm{RD}} \bar{\gamma}_{\mathrm{RR}}}\right)+e^{-\frac{\overline{\bar{R}}_{\mathrm{RD}} k+\bar{\gamma}_{\mathrm{SR}}}{\overline{\mathrm{\gamma}}_{\mathrm{RD}} \bar{\gamma}_{\mathrm{RR}}}} \sum_{j=1}^{i}\left(\frac{\bar{\gamma}_{\mathrm{RR}} \bar{\gamma}_{\mathrm{RD}_{1}}}{\bar{\gamma}_{\mathrm{RD}_{1}} k+\bar{\gamma}_{\mathrm{SR}}}\right)(-1)^{j-1}(j-1) !\right)\right]\right\} .
\end{aligned}
$$

$$
\begin{aligned}
\bar{C}_{2} \approx & \frac{1}{\ln 2} \sum_{k=0}^{K}\left(\begin{array}{c}
K \\
k
\end{array}\right)(-1)^{k}\left(\frac{a_{2} \bar{\gamma}_{\mathrm{SR}}}{a_{2} \bar{\gamma}_{\mathrm{SR}}-\bar{\gamma}_{\mathrm{RR}}}\right)^{k} e^{\frac{\bar{\gamma}_{\mathrm{RD}} k+\bar{\gamma}_{\mathrm{SR}}}{a_{2} \bar{\gamma}_{\mathrm{SR}} \overline{\mathrm{RD}}_{2}}}\left[\operatorname{Ei}\left(-\frac{\bar{\gamma}_{\mathrm{RD}} k+\bar{\gamma}_{\mathrm{SR}}}{a_{2} \bar{\gamma}_{\mathrm{SR}} \bar{\gamma}_{\mathrm{RD}_{2}}}\right)+\sum_{i=0}^{k-1} \frac{e^{\xi_{3}}}{\xi_{3}^{-i}} \frac{(-1)^{i+1}}{i !}\left(\operatorname{Ei}\left(-\frac{\bar{\gamma}_{\mathrm{RD}} k+\bar{\gamma}_{\mathrm{SR}}}{\bar{\gamma}_{\mathrm{RD}_{2}} \bar{\gamma}_{\mathrm{RR}}}\right)\right.\right. \\
& \left.\left.+e^{-\frac{\bar{\gamma}_{\mathrm{RD}} k+\bar{\gamma}_{\mathrm{SR}}}{\bar{\gamma}_{\mathrm{RD}} \bar{\gamma}_{\mathrm{RR}}}} \sum_{j=1}^{i}\left(\frac{\bar{\gamma}_{\mathrm{RD}_{2}} \bar{\gamma}_{\mathrm{RR}}}{\bar{\gamma}_{\mathrm{RD}_{2}} k+\bar{\gamma}_{\mathrm{SR}}}\right)^{j}(-1)^{j-1}(j-1) !\right)\right]-\frac{1}{\ln 2} e^{-\frac{1}{a_{2} \bar{\gamma}_{\mathrm{RD}_{2}}}} \operatorname{Ei}\left(-\frac{1}{a_{2} \bar{\gamma}_{\mathrm{RD}_{2}}}\right) .
\end{aligned}
$$

Proof. See Appendix C.

\section{B. Ergodic capacity at $\mathrm{D}_{2}$}

The instantaneous capacity at the strong user $\mathrm{D}_{2}$ is given as

$$
C_{2}=\log _{2}\left(1+\gamma_{\mathrm{D}_{2}}\right),
$$

where $\gamma_{D_{2}}$ is the instantaneous received E2E SINR, given as in (12). Therefore, the ergodic capacity at $\mathrm{D}_{2}$, defined as the expectation of the expression in (36), can be given as

$$
\begin{aligned}
\bar{C}_{2} & =\mathrm{E}\left[\log _{2}\left(1+\gamma_{\mathrm{D}_{2}}\right)\right] \\
& =\frac{1}{\ln 2} \int_{0}^{\infty} \frac{1-\operatorname{Pr}\left(\gamma_{\mathrm{D}_{2}}<x\right)}{1+x} \mathrm{~d} x .
\end{aligned}
$$

Then, an approximate expression for the ergodic capacity at $\mathrm{D}_{2}$ can be attained from (37) in closed form, as in the following proposition.

Proposition 8. In a relay-aided NOMA system with two destinations, in which PRS among $K$ FD-AF relays is employed, an approximated closed-form expression for the ergodic capacity of the strong user, $\mathrm{D}_{2}$, is given by (33), shown at the top of this page, where

$$
\xi_{3}=\frac{\left(a_{2} \bar{\gamma}_{\mathrm{SR}}-\bar{\gamma}_{\mathrm{RR}}\right)\left(k \bar{\gamma}_{\mathrm{RD}_{2}}+\bar{\gamma}_{\mathrm{SR}}\right)}{\left(a_{2} \bar{\gamma}_{\mathrm{SR}} \bar{\gamma}_{\mathrm{RR}} \bar{\gamma}_{\mathrm{RD}_{2}}\right)} .
$$

Proof. See Appendix D.

\section{NUMERICAL RESULTS AND DISCUSSIONS}

In this section, illustrative examples are considered to assess the impact of the key system parameters on both the outage probability and the ergodic capacity of users $\mathrm{D}_{1}$ and $\mathrm{D}_{2}$. Moreover, the analytical expressions obtained in Sections III and IV, respectively, are validated through Monte Carlo simulations. For this purpose, let us consider a linear topology in which the normalized distances of the links $\mathrm{S} \rightarrow \mathrm{D}_{\ell}, \mathrm{S} \rightarrow \mathrm{R}_{k}, \mathrm{R}_{k} \rightarrow \mathrm{D}_{\ell}$ are set to $d_{\mathrm{SD}_{1}}=1, d_{\mathrm{SD}_{2}}=0.75, d_{\mathrm{SR}_{k}}=0.5, d_{\mathrm{R}_{k} \mathrm{D}_{1}}=0.5$

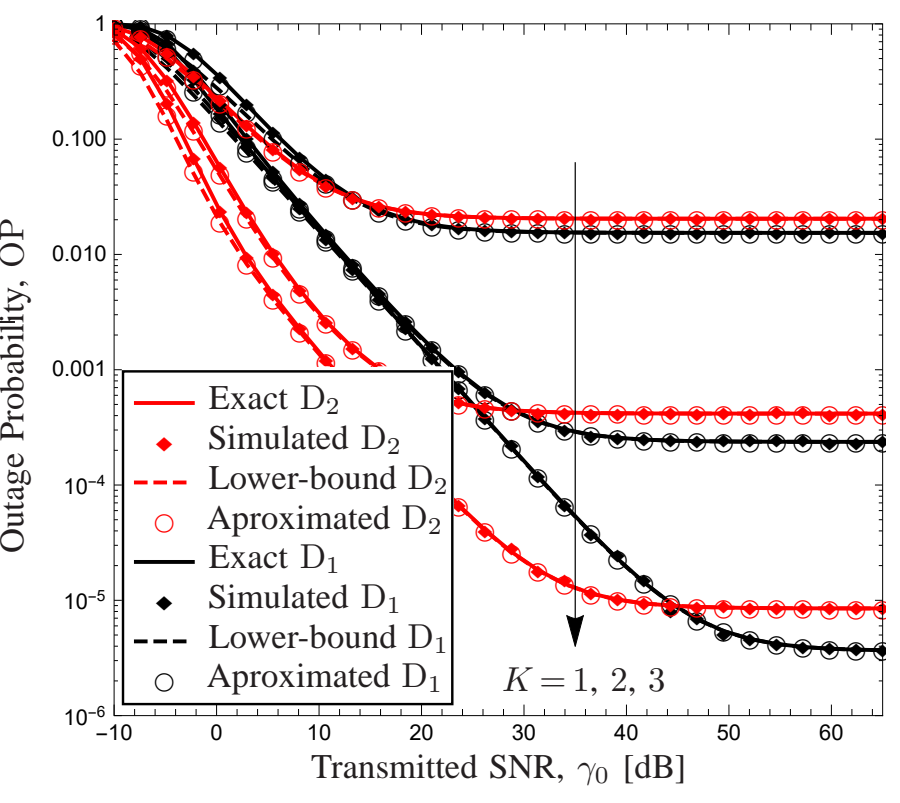

Fig. 2. Outage probability of NOMA users, $D_{1}$ and $D_{2}$, versus transmit SNR for a different number of relays $K=1,2$ and 3 , considering an average SI channel gain $\Omega_{\mathrm{RR}}=-10 \mathrm{~dB}$ and power allocation factors $\left(a_{1}, a_{2}\right)=$ $(0.7,0.3)$.

and $d_{\mathrm{R}_{k} \mathrm{D}_{2}}=0.25$. In addition, we consider that the average channel gains of all links are determined by the path loss, that is, $\Omega_{A}=d_{A}^{-\eta}$, with $A \in\left\{\mathrm{SR}_{k}, \mathrm{R}_{k} \mathrm{D}_{\ell}\right\}$, where $d_{A}$ is the distance between two nodes and $\eta$ is the path-loss exponent. In the following examples, the path-loss exponent is set to $\eta=4$ and the SNR threshold is set to $\tau=0 \mathrm{~dB}$.

Fig. 2 shows the outage performance of the NOMA destinations, $\mathrm{D}_{1}$ and $\mathrm{D}_{2}$, as a function of the transmit SNR for different values of the number of relays $K=1,2,3$. The power allocation factors for users $\mathrm{D}_{1}$ and $\mathrm{D}_{2}$ are set to $a_{1}=0.7$ and $a_{2}=0.3$, respectively, and the average channel gain at the SI link is considered to be $\Omega_{\mathrm{RR}}=-10 \mathrm{~dB}$. From Fig. 2, note 


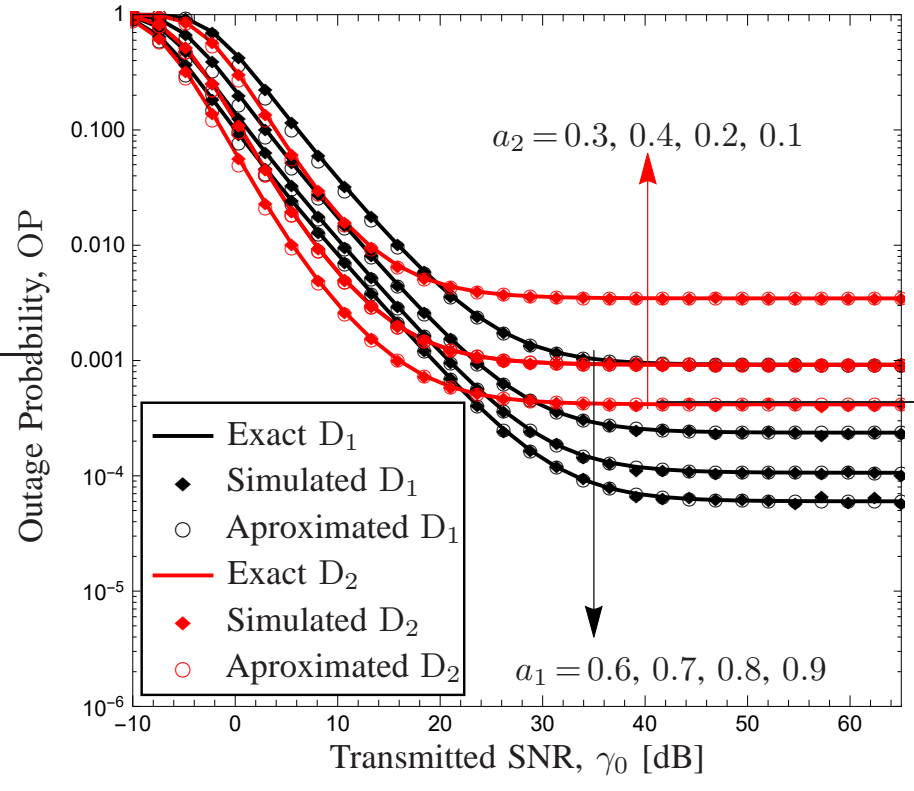

Fig. 3. Outage probability of NOMA users, $D_{1}$ and $D_{2}$, versus transmit SNR for different values of the power allocation factor $\left(a_{1}, a_{2}\right)=(0.6,0.4)$, $(0.7,0.3),(0.8,0.2)$, and $(0.9,0.1)$, considering an average SI channel gain $\Omega_{\mathrm{RR}}=-10 \mathrm{~dB}$ and a number of relays $K=2$.

that our expressions derived from the exact analysis, given in (16) and (18), perfectly match with Monte Carlo simulations. Note also that the approximate expressions obtained in (24) and (26) are very tight to the exact curves, along the whole range of SNR values. In addition, the lower-bound expressions attained in (19) and (21) result in excellent approximations to the exact curves in the medium-to-high SNR regime. We can observe that the outage performance curves saturate in a floor at the high SNR regime, thus resulting in a system diversity order equal to zero, as can be corroborated from the asymptotic analytical expressions obtained in Section III-D. Such a value for the system diversity order arises from the residual SI detrimental effect at the FD relays, which worsens as the transmit power increases, thereby preventing the system performance from improving. On the other hand, we can observe that as the number of relays increases, the outage performance of both users improves, as expected. However, at low SNR, increasing the number of relays above $K=2$, does renders an improvement on the outage performance at the weak user, $\mathrm{D}_{1}$, it does not render any improvement, thus requiring higher values of transmit SNR to take advantage of the cooperative diversity for this user.

In Fig. 3, the outage probability for both NOMA destinations is illustrated as a function of the transmit SNR for different values of power allocation factors, namely, $a_{1}=0.6,0.7,0.8,0.9$, with $a_{2}=1-a_{1}$. In this case, we consider that $K=2$ and $\Omega_{\mathrm{RR}}=-10 \mathrm{~dB}$. It can be observed that the weak user $\mathrm{D}_{1}$ always outperforms the strong user $\mathrm{D}_{2}$, regardless of the power allocation factors, as long as the condition $a_{1}>a_{2}$ is satisfied. More specifically, as $a_{1}$ increases and, consequently, $a_{2}$ decreases, the outage performance of weak user $\mathrm{D}_{1}$ gradually improves, whereas that of strong user $\mathrm{D}_{2}$ deteriorates. Note that, for $\left(a_{1}, a_{2}\right)=(0.6,0.4)$, the

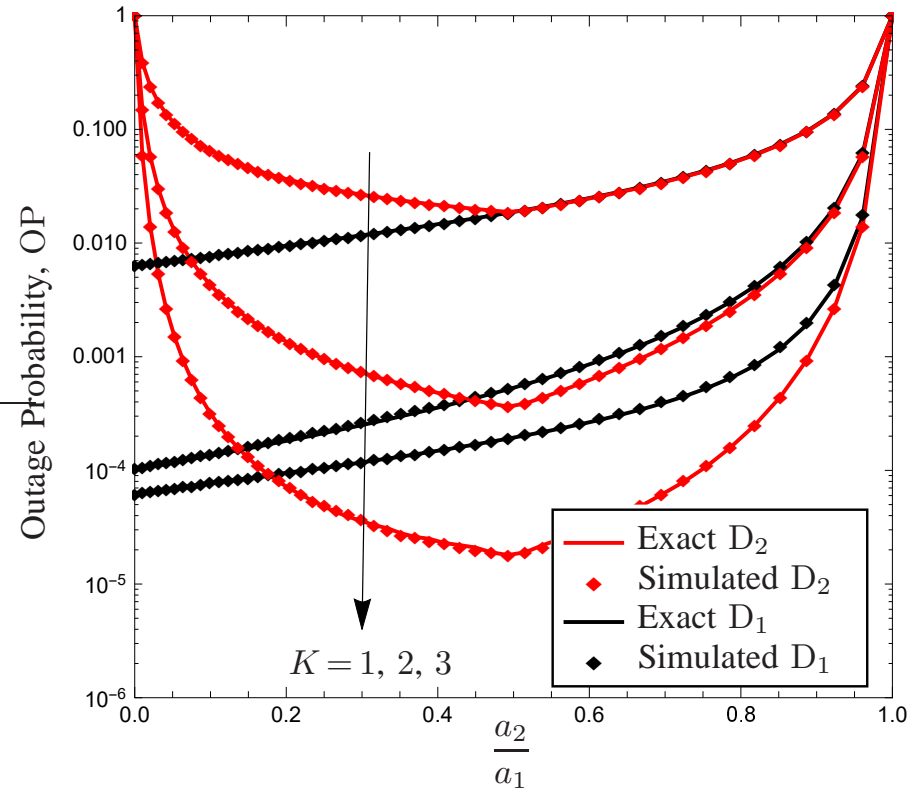

Fig. 4. Outage probability of NOMA users, $D_{1}$ and $D_{2}$, versus the power allocations ratio $a_{2} / a_{1}$ for a different number of relays $K=1,2$ and 3 , considering an average SI channel gain $\Omega_{\mathrm{RR}}=-10 \mathrm{~dB}$ and transmit SNR, $\gamma_{0}=30 \mathrm{~dB}$.

outage performance of both users is almost equivalent. On the other hand, it is noteworthy that from the point where the condition $a_{2} \geq a_{1} /(\tau+1)^{6}$ is met, for lower values of $a_{1}$ and, consequently, higher values of $a_{2}$-for example, $\left(a_{1}, a_{2}\right)=(0.55,0.45)$ - not only the outage performance of the weak user $\mathrm{D}_{1}$, but also that of strong user $\mathrm{D}_{2}$ worsens ${ }^{7}$. This can be explained by the fact that the strong user first needs to decode the weak user's information through a SIC technique, so that the strong user is able to decode its own information. Thus, as $a_{1}$ decreases and $a_{2}$ increases, the received SINR at $D_{2}$ relative to $D_{1}$ 's information diminishes, such that SIC at $\mathrm{D}_{2}$ fails, as can be corroborated from (11) and (17). As a result, the strong user $D_{2}$ is also unable to decode its own information. Note also that, for this illustrative scenario, the outage performance of the strong user $D_{2}$ is the same for $a_{2}=0.2$ and 0.4 .

To complement the above discussion, Fig. 4 shows the outage probability of the NOMA destinations, $\mathrm{D}_{1}$ and $\mathrm{D}_{2}$, as a function of the ratio $a_{2} / a_{1}$ for different values of the number of relays $K=1,2,3$. In this case, the average channel gain at the SI link is set to $\Omega_{\mathrm{RR}}=-10 \mathrm{~dB}$ and the transmit SNR is fixed at $\gamma_{0}=30 \mathrm{~dB}$. Recall that, according to the NOMA scheme for our scenario, the following relationships for the power allocation factors must be satisfied: $a_{1}+a_{2}=1$ and $a_{2}<a_{1}$. Note that as the number of relays increases, the outage performance of both destinations improves, as expected. On the other hand, note that, for the weak user $\mathrm{D}_{1}$, the outage performance worsens as the ratio $a_{2} / a_{1}$ increases,

${ }^{6}$ This relationship is obtained by comparing the analytical expressions for the asymptotic outage probability at $D_{1}$ and $D_{2}$ attained in (28) and (29), respectively, and from the definitions of $\widetilde{\tau}$ and $\theta$ introduced in Propositions 1 and 2.

${ }^{7}$ Notice that, for clarity purposes, the results corresponding to these cases, such as $\left(a_{1}, a_{2}\right)=(0.55,0.45)$, were omitted from Fig. 3 . 


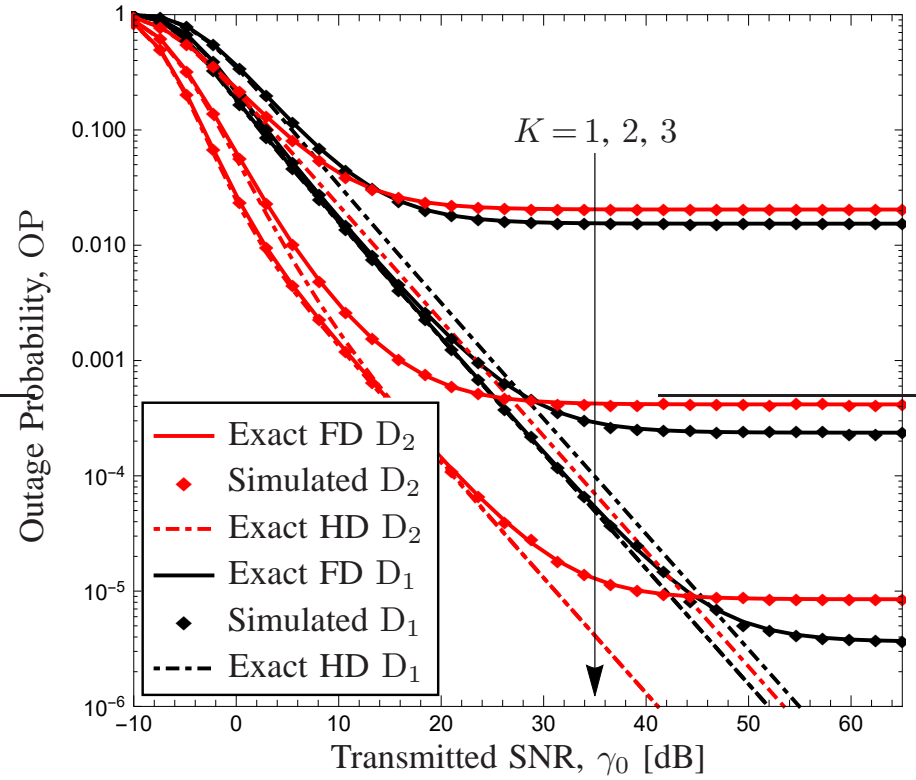

Fig. 5. Comparison of the outage probability of users $D_{1}$ and $D_{2}$ between FD- and HD-based NOMA schemes, considering a power allocation factor $\left(a_{1}, a_{2}\right)=(0.7,0.3)$, different values of the number of relays $K=1,2,3$, and an average SI channel gain $\Omega_{\mathrm{RR}}=-10 \mathrm{~dB}$.

which is intuitive since less power is allocated to $\mathrm{D}_{1}$. Meanwhile, we can observe that, for the strong user $\mathrm{D}_{2}$, the outage probability improves as $a_{2} / a_{1}$ increases, until the condition $a_{2} / a_{1} \geq 1 /(\tau+1)$ is met, as previously stated. From this point (in this case, for $\tau=0 \mathrm{~dB}$, from $a_{2} / a_{1}=0.5$ ), the outage probability of $\mathrm{D}_{2}$ also worsens, since the SIC process at $\mathrm{D}_{2}$ to decode the information relative to $\mathrm{D}_{1}$ fails more frequently, such that $\mathrm{D}_{2}$ is no longer able to decode its own information.

Fig. 5 illustrates a comparison of the outage probability for the NOMA users $D_{1}$ and $D_{2}$ versus the transmit SNR between the FD-based scheme analyzed herein and the HDbased counterpart proposed in [14]. To do so, we consider our exact analytical expressions derived in Section III-A and those corresponding to the HD-based scheme, presented in [14, eqs. (25) and (29)]. In this case, the following system parameters are taken into account: number of relays $K=1,2,3$, power allocation factors $\left(a_{1}, a_{2}\right)=(0.7,0.3)$, and average SI channel gain $\Omega_{R R}=-10 \mathrm{~dB}$. From this figure, we can observe that, unlike the proposed FD-based scheme, the outage performance of the HD-based counterpart does not saturates at high SNR, as expected-since HD relays do not suffer from SI. However, it is worth pointing out that the HD-based scheme requires two time slots to transmit information from the source to the NOMA destinations, thus halving the system spectral efficiency with respect to the proposed FD-based NOMA scheme. Moreover, at low-to-medium SNR, the FDbased scheme presents the same performance as that obtained by the HD-based one, even though a conservative value for SI attenuation was considered $\left(\Omega_{\mathrm{RR}}=-10 \mathrm{~dB}\right)$. Note also that as the number of relays increases the outage performance of HD-based scheme slightly improves. Hence, the proposed scheme is more suitable for the low-to-medium SNR regime

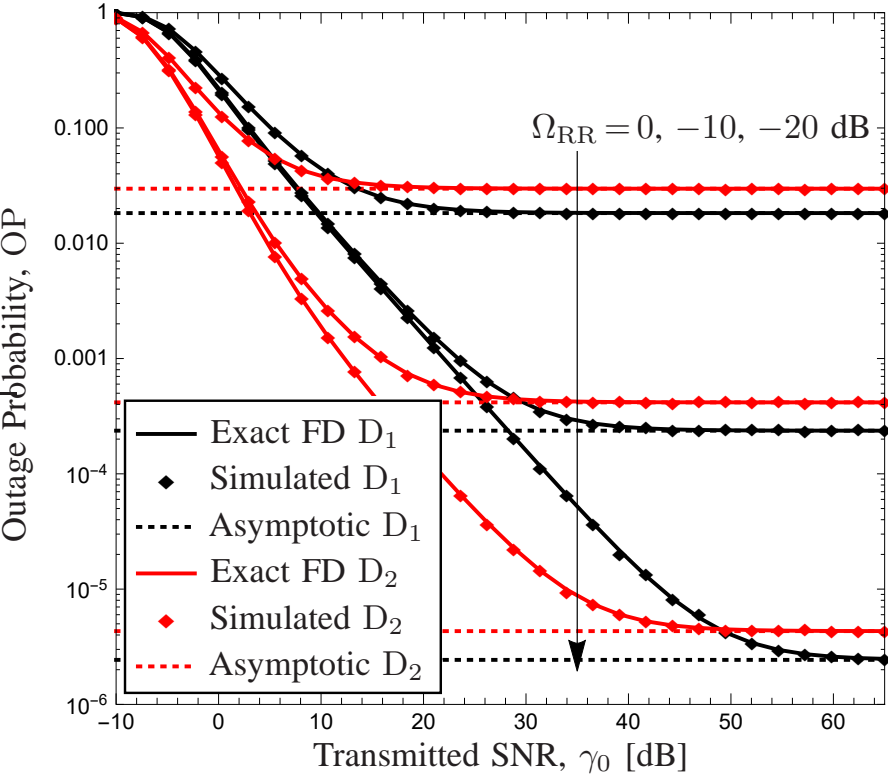

Fig. 6. Outage probability of NOMA users, $\mathrm{D}_{1}$ and $\mathrm{D}_{2}$, versus transmit SNR for different values of average SI channel gain $\Omega_{\mathrm{RR}}=0,-10,-20 \mathrm{~dB}$, considering power allocation factors $\left(a_{1}, a_{2}\right)=(0.7,0.3)$ and a number of relays $K=2$.

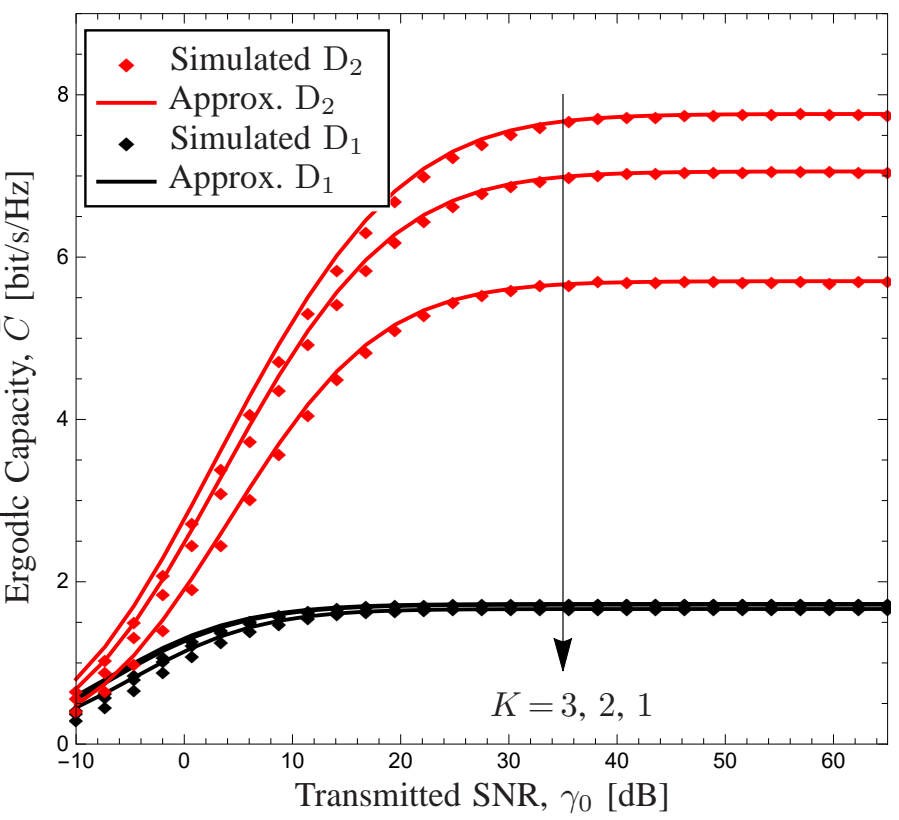

Fig. 7. Ergodic capacity of NOMA users, $\mathrm{D}_{1}$ and $\mathrm{D}_{2}$, versus transmit SNR for a different number of relays $K=1,2$ and 3 , considering an average SI channel gain $\Omega_{\mathrm{RR}}=-10 \mathrm{~dB}$ and power allocation factors $\left(a_{1}, a_{2}\right)=$ $(0.7,0.3)$.

with the advantage of an improved spectral efficiency.

Fig. 6 shows the outage probability of the NOMA destinations, $\mathrm{D}_{1}$ and $\mathrm{D}_{2}$, versus the transmit $\mathrm{SNR}$ for different values of average SI channel gain $\Omega_{\mathrm{RR}}=0,-10,-20 \mathrm{~dB}$, by considering $K=2$ and $\left(a_{1}, a_{2}\right)=(0.7,0.3)$. Note from this figure that as $\Omega_{\mathrm{RR}}$ decreases, the outage floor level also decreases and occurs at higher transmit SNR values. Additionally, note that the curves obtained from the asymptotic expressions given in (28) and (29) perfectly match the performance floor level at 
high SNR, thus validating the asymptotic analysis performed from the lower-bound expressions of the outage probability.

Fig. 7 shows the ergodic capacity of the NOMA destinations $D_{1}$ and $D_{2}$ versus the transmitted SNR for different values of the number of relays $K=1,2,3$. The power allocation factors for the users $D_{1}$ and $D_{2}$ are respectively set to $a_{1}=0.7$ and $a_{2}=0.3$, and the average channel gain at the SI link is set to $\Omega_{\mathrm{RR}}=-10 \mathrm{~dB}$. We can observe from Fig. 7 that the analytical expressions obtained in (32) and (33) prove to be excellent approximations to the true performance for the entire range of SNR values. Note that the ergodic capacity for users $\mathrm{D}_{1}$ and $\mathrm{D}_{2}$ also saturate to a floor at the high SNR regime due to the detrimental effect of residual SI at the FD relays. Note also that, while the ergodic capacity for the strong user $\mathrm{D}_{2}$ improves as the number of relays increases, the ergodic capacity for the weak user $\mathrm{D}_{1}$ does not present a significant improvement with an increment of the number of relays.

Fig. 8 illustrates the system sum rate versus the transmit SNR, as well as a comparison with OMA-based cooperative systems in which PRS among either multiple HD-AF relays or multiple FD-AF relays is employed ${ }^{8}$. In this case, the following system parameters are considered: number of relays $K=1,2,3$, power allocation factors $\left(a_{1}, a_{2}\right)=(0.7,0.3)$ and normalized distance of the links $\mathrm{R}_{k} \rightarrow \mathrm{D}_{\text {OMA }}, d_{\mathrm{R}_{k} \mathrm{D}_{\text {OMA }}}=0.5$. Note that the sum rate of the proposed NOMA system outperforms OMA-based systems at low-to-medium SNR. This agrees with the results obtained in Fig. 5 for the outage probability, thus showcasing the benefits of the joint use of NOMA and FD relaying techniques at this region. Nevertheless, we can notice that the performance of the proposed NOMA system saturates due the residual SI at the FD relays, thus yielding a lower sum rate with respect to the HD-OMA system and resulting in a equal sum rate with respect to the FD-OMA system, at high SNR.

\section{CONCLUSIONS}

This paper investigated the outage and ergodic capacity performance of a relay-aided NOMA system with two destinations, in which PRS among multiple FD-AF relays is employed. We derived exact analytical expressions for the outage probability and ergodic capacity of the strong user and the weak user. With the aim of reducing the computational complexity, closed-form expressions for the outage probability were obtained from both approximation- and lower boundbased analyses. In addition, closed-form approximations for the ergodic capacity of each NOMA destination and for the system sum rate were derived. Monte Carlo simulations were provided to validate the accuracy of our formulas. Numerical results showed that an outage floor occurs due to the inherent

\footnotetext{
${ }^{8}$ For both HD-OMA and FD-OMA systems, the network setup consists of one source, $K$ relays, and two destinations, all operating on a TDMA basis, as in the FD-NOMA system considered herein. For the HD-OMA system, the source is assumed to send a message to each destination within one different fading block, which is divided into two time slots (one for the source-relay transmission and one for the relay-destination transmission). Thus, the overall communication process from the source to the two destinations takes four time slots. In the FD-OMA system, the system sum rate is doubled with respect to that of HD-OMA system, as different messages are transmitted in each one of the two time slots composing a fading block due to FD relaying operation.
}

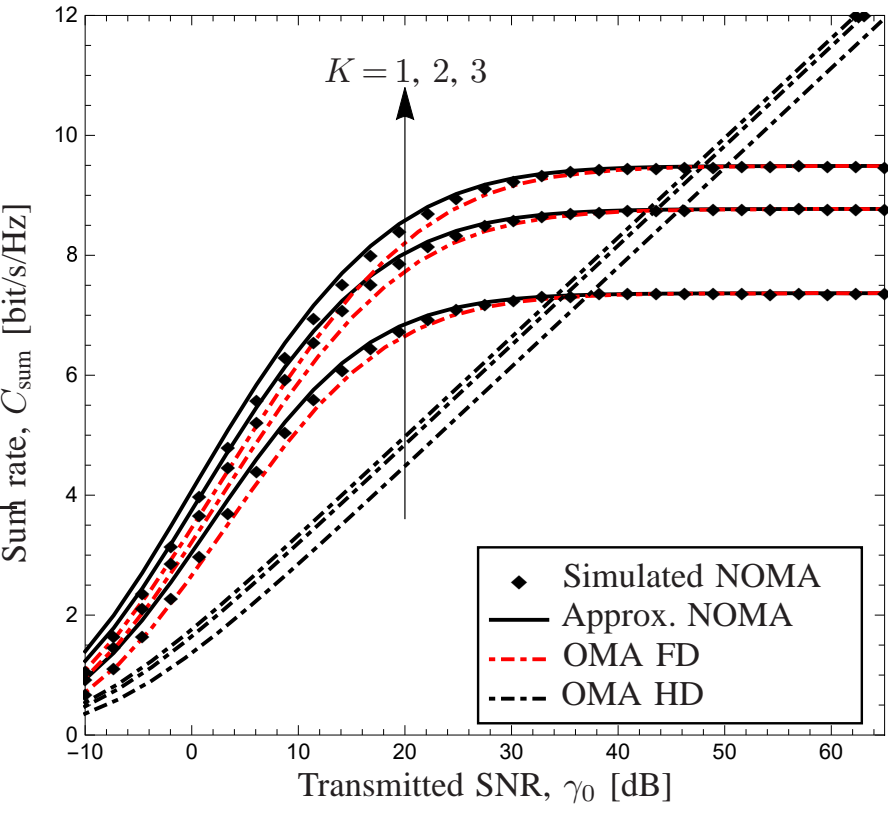

Fig. 8. System sum rate versus transmit SNR for a different number of relays $K=1,2$ and 3 , considering an average SI channel gain $\Omega_{\mathrm{RR}}=-10 \mathrm{~dB}$ and power allocation factors $\left(a_{1}, a_{2}\right)=(0.7,0.3)$.

SI of FD operation at the relays, thus rendering the system diversity order zero. Asymptotic closed-form expressions were attained at the high SNR regime, which characterize the outage floor level in terms of the system parameters. This performance floor level can be reduced whether the number of relays is increased or the average SI channel gain is diminished (or, equivalently, the SI attenuation is improved, thus reinforcing the importance of highly effective SI cancellation techniques). Nevertheless, the outage performance of the proposed FDbased scheme showed to be equivalent to its HD-based counterpart at low-to-medium SNR, even for conservative values of the average SI channel gain. Moreover, the system sum rate of the proposed FD-based scheme presents the advantage of spectral efficiency with respect to the OMA-based schemes, especially at low-to-medium SNR. Additionally, it was established that increasing the power allocation factor of the strong user resulted in a performance improvement, until some value from which the outage performance of both NOMA users worsens, since the correct decoding of strong user' message depends on the success of the SIC process relative to the weak user's information.

Some interesting extensions for future development of this investigation is to study optimal/suboptimal relay selection strategies and optimal power allocation schemes, such as those proposed in $[18,19]$ for HD-relay aided NOMA scenarios, as well as to assess the impact of imperfect SIC on the system performance $[22,32]$.

APPENDIX A

\section{ProOF OF PROPOSITION 1}

In the following, we derive the exact outage probability for weak user $\mathrm{D}_{1}$. From (15), by using (14), the outage probability 
for $\mathrm{D}_{1}$ can be obtained as

$$
\begin{aligned}
& \mathrm{OP}_{1}=\operatorname{Pr}\left(\frac{a_{1} Z_{k^{*}} Y_{k^{*} 1}}{a_{2} Z_{k^{*}} Y_{k^{*} 1}+Z_{k^{*}}+Y_{k^{*} 1}+1}<\tau\right) \\
& =\operatorname{Pr}\left(Z_{k^{*}}\left(Y_{k^{*} 1}\left(a_{1}-a_{2} \tau\right)-\tau\right)<\tau\left(Y_{k^{*} 1}+1\right)\right) \\
& =\operatorname{Pr}\left(Z_{k^{*}}<\frac{\widetilde{\tau}\left(Y_{k^{*} 1}+1\right)}{Y_{k^{*} 1}-\widetilde{\tau}}\right) \\
& \stackrel{(b)}{=} \sum_{k=1}^{K}\left[\operatorname{Pr}\left(Z_{k}<\frac{\widetilde{\tau}\left(Y_{k 1}+1\right)}{Y_{k 1}-\widetilde{\tau}} \mid k^{*}=k\right) \operatorname{Pr}\left(k^{*}=k\right)\right] \\
& =\sum_{k=1}^{K}\left[\operatorname{Pr}\left(Z_{k}<\frac{\widetilde{\tau}\left(Y_{k 1}+1\right)}{Y_{k 1}-\widetilde{\tau}} \mid k^{*}=k\right) \prod_{\substack{j=1 \\
j \neq k}}^{K} \operatorname{Pr}\left(Z_{j}<Z_{k}\right)\right] \\
& =\sum_{k=1}^{K}\left[\int_{0}^{\infty} \operatorname{Pr}\left(Z_{k}<\frac{\widetilde{\tau}\left(y_{1}+1\right)}{y_{1}-\tilde{\tau}} \mid k^{*}=k\right)\right. \\
& \left.\times \prod_{\substack{j=1 \\
j \neq k}}^{K} \operatorname{Pr}\left(Z_{j}<Z_{k}\right) f_{Y_{k 1}}\left(y_{1}\right) \mathrm{d} y_{1}\right] \\
& =\sum_{k=1}^{K} \int_{0}^{\widetilde{\tau}} \prod_{\substack{j=1 \\
j \neq k}}^{K}\left[\operatorname{Pr}\left(Z_{j}<Z_{k}\right)\right] f_{Y_{k 1}}\left(y_{1}\right) \mathrm{d} y_{1} \\
& +\sum_{k=1}^{K} \int_{\widetilde{\tau}}^{\infty} \operatorname{Pr}\left(Z_{k}<\frac{\widetilde{\tau}\left(y_{1}+1\right)}{y_{1}-\widetilde{\tau}} \mid k^{*}=k\right) \\
& \times \prod_{\substack{j=1 \\
j \neq k}}^{K}\left[\operatorname{Pr}\left(Z_{j}<Z_{k}\right)\right] f_{Y_{k 1}}\left(y_{1}\right) \mathrm{d} y_{1} \\
& =\sum_{k=1}^{K} \int_{0}^{\widetilde{\tau}} \int_{0}^{\infty} \prod_{\substack{j=1 \\
j \neq k}}^{K}\left[F_{Z_{j}}(z)\right] f_{Z_{k}}(z) f_{Y_{k 1}}\left(y_{1}\right) \mathrm{d} z \mathrm{~d} y_{1} \\
& +\sum_{k=1}^{K} \int_{\widetilde{\tau}}^{\infty} \int_{0}^{\infty} F_{Z_{k}}\left(\frac{\widetilde{\tau}\left(y_{1}+1\right)}{y_{1}-\widetilde{\tau}}\right) \\
& \times \prod_{\substack{j=1 \\
j \neq k}}^{K}\left[F_{Z_{j}}(z)\right] f_{Z_{k}}(z) f_{Y_{k 1}}\left(y_{1}\right) \mathrm{d} z \mathrm{~d} y_{1} \\
& \stackrel{(c)}{=} K\left[\int_{0}^{\widetilde{\tau}} \int_{0}^{\infty}\left[F_{Z}(z)\right]^{K-1} f_{Z}(z) f_{Y_{1}}\left(y_{1}\right) \mathrm{d} z \mathrm{~d} y_{1}\right] \\
& +K\left[\int_{\widetilde{\tau}}^{\infty} \int_{0}^{\frac{\widetilde{\tau}\left(y_{1}+1\right)}{y_{1}-\widetilde{\tau}}}\left[F_{Z}(z)\right]^{K-1} f_{Z}(z) f_{Y_{1}}\left(y_{1}\right) \mathrm{d} z \mathrm{~d} y_{1}\right] \\
& =K\left[\int_{0}^{\widetilde{\tau}} \int_{0}^{\infty}\left[F_{Z}(z)\right]^{K-1} f_{Y_{1}}\left(y_{1}\right) \mathrm{d} F_{Z} \mathrm{~d} y_{1}\right] \\
& +K\left[\int_{\widetilde{\tau}}^{\infty} \int_{0}^{\frac{\widetilde{\tau}\left(y_{1}+1\right)}{y_{1}-\widetilde{\tau}}}\left[F_{Z}(z)\right]^{K-1} f_{Y_{1}}\left(y_{1}\right) \mathrm{d} F_{Z} \mathrm{~d} y_{1}\right] \\
& =F_{Y_{1}}(\widetilde{\tau})+\int_{\widetilde{\tau}}^{\infty}\left[F_{Z}\left(\frac{\left(y_{1}+1\right) \widetilde{\tau}}{y_{1}-\widetilde{\tau}}\right)\right]^{K} f_{Y_{1}}\left(y_{1}\right) \mathrm{d} y_{1} \text {, }
\end{aligned}
$$

where step $(b)$ follows from applying the Total Probability Theorem [34], and step $(c)$ follows from considering i.i.d. fading channels. Furthermore, $F_{Y_{1}}\left(y_{1}\right)=1-\exp \left(-y_{1} / \bar{\gamma}_{\mathrm{RD}_{1}}\right)$ and $f_{Y_{1}}\left(y_{1}\right)=\left(1 / \bar{\gamma}_{\mathrm{RD}_{1}}\right) \exp \left(-y_{1} / \bar{\gamma}_{\mathrm{RD}_{1}}\right)$ are the $\mathrm{CDF}$ and PDF of an exponential RV with mean value $\bar{\gamma}_{\mathrm{RD}_{1}}$, respectively. Besides, the CDF of $Z_{k}$ can be determined as

$$
\begin{aligned}
F_{Z}(z) & =\operatorname{Pr}\left(\frac{X_{k}}{U_{k}+1}<z\right) \\
& =\int_{0}^{\infty} F_{X}(z(u+1)) f_{U}(u) \mathrm{d} u \\
& =1-\frac{e^{-\frac{z}{\bar{\gamma}_{\mathrm{SR}}}}}{1+\left(\bar{\gamma}_{\mathrm{RR}} / \bar{\gamma}_{\mathrm{SR}}\right) z},
\end{aligned}
$$

where $F_{X}(x)=1-\exp \left(-x / \bar{\gamma}_{\mathrm{SR}}\right)$ and $f_{U}(u)=$ $\left(1 / \bar{\gamma}_{\mathrm{RR}}\right) \exp \left(-u / \bar{\gamma}_{\mathrm{RR}}\right)$ are the CDF and PDF of exponential RVs with mean values $\bar{\gamma}_{\mathrm{SR}}$ and $\bar{\gamma}_{\mathrm{RR}}$, respectively. Then, after substituting (40) into (39), the exact outage probability for the weak user, $\mathrm{D}_{1}$, can be obtained as in (16).

\section{APPENDIX B}

\section{PROOF OF PROPOSITION 2}

Next we derive the exact outage probability of strong user $\mathrm{D}_{2}$. From (17), by considering the definition of $Z_{k}$ in (14) and following a procedure similar to the proof of Proposition 1, the outage probability for $\mathrm{D}_{2}$ can be developed as

$$
\begin{aligned}
\mathrm{OP}_{2}= & \operatorname{Pr}\left(\frac{a_{1} Z_{k^{*}} Y_{k^{*} 2}}{a_{2} Z_{k^{*}} Y_{k^{*} 2}+Z_{k^{*}}+Y_{k^{*} 2}+1}<\tau\right) \\
& +\operatorname{Pr}\left(\frac{a_{2} Z_{k^{*}} Y_{k^{*} 2}}{Z_{k^{*}}+Y_{k^{*} 2}+1}<\tau, \frac{a_{1} Z_{k^{*}} Y_{k^{*} 2}}{a_{2} Z_{k^{*}} Y_{k^{*} 2}+Z_{k^{*}}+Y_{k^{*} 2}+1}>\tau\right) \\
\stackrel{(d)}{=} & \operatorname{Pr}\left(\frac{Z_{k^{*} Y_{k^{*} 2}}}{Z_{k^{*}}+Y_{k^{*} 2}+1}<\widetilde{\tau}\right)+\operatorname{Pr}\left(\frac{Z_{k^{*}} Y_{k^{*} 2}}{Z_{k^{*}}+Y_{k^{*} 2}+1}<\tau^{\prime}\right) \\
& -\operatorname{Pr}\left(\frac{Z_{k^{*} Y_{k^{*} 2}}}{Z_{k^{*}}+Y_{k^{*} 2}+1}<\widetilde{\tau}, \frac{Z_{k^{*}} Y_{k^{*} 2}}{Z_{k^{*}}+Y_{k^{*} 2}+1}<\tau^{\prime}\right) \\
& \stackrel{(e)}{=} \operatorname{Pr}\left(\frac{Z_{k^{*}} Y_{k^{*} 2}}{Z_{k^{*}}+Y_{k^{*} 2}+1}<\max \left\{\widetilde{\tau}, \tau^{\prime}\right\} \triangleq \theta\right) \\
= & \operatorname{Pr}\left(Z_{k^{*}}<\frac{\left(Y_{k^{*} 2}+1\right) \theta}{Y_{k^{*} 2}-\theta}\right),
\end{aligned}
$$

where step $(d)$ follows from applying the identity $\operatorname{Pr}(A, B)=\operatorname{Pr}(A)-\operatorname{Pr}(A, \bar{B})$ [34]; and step (e) follows from analyzing the probability of the joint event at the third term in step $(d)$, regarding the relationships $\tilde{\tau}>\tau^{\prime}$ and $\tau^{\prime}>\widetilde{\tau}$, from which we can note that the occurrence of the event related to the lower value between $\widetilde{\tau}$ and $\tau^{\prime}$ entails the occurrence of the other event, so that the overall outage probability at $\mathrm{D}_{2}$ is determined by $\max \left\{\widetilde{\tau}, \tau^{\prime}\right\}$. From (41), by following a similar procedure to that presented in Proposition $1, \mathrm{OP}_{2}$ can be expressed as

$$
\mathrm{OP}_{2}=F_{Y_{2}}(\theta)+\int_{\theta}^{\infty}\left[F_{Z}\left(\frac{\left(y_{2}+1\right) \theta}{y_{2}-\theta}\right)\right]^{K} f_{Y_{2}}\left(y_{2}\right) \mathrm{d} y_{2},
$$

where $F_{Y_{2}}\left(y_{2}\right)=1-\exp \left(-y_{2} / \bar{\gamma}_{\mathrm{RD}_{2}}\right)$ and $f_{Y_{2}}\left(y_{2}\right)=$ $\left(1 / \bar{\gamma}_{\mathrm{RD}_{2}}\right) \exp \left(-y_{2} / \bar{\gamma}_{\mathrm{RD}_{2}}\right)$ are the CDF and PDF of an exponential RV with mean value $\bar{\gamma}_{\mathrm{RD}_{2}}$, respectively, and $F_{Z}(\cdot)$ is given as in (40). Then, after changing the limits of the integral in (42), an exact analytical expression for the outage probability of the strong user, $\mathrm{D}_{2}$, can be obtained as in (18). 


\section{APPENDIX C \\ PROOF OF PROPOSITION 7}

In the following, we derive the ergodic capacity for weak user $\mathrm{D}_{1}$. By substituting (15) considering the definition of $Z_{k}$ given in (14), the ergodic capacity for $\mathrm{D}_{1}$ can be obtained as

$$
\begin{aligned}
& \bar{C}_{1}=\frac{1}{\ln 2} \int_{0}^{\infty} \frac{1-\operatorname{Pr}\left(\frac{a_{1} Z_{k^{*}} Y_{k^{*}}}{\left.a_{2} Z_{k^{*} Y_{k^{*}}+Z_{k^{*}}+Y_{k^{*} 1}+1}<x\right)}\right.}{1+x} \mathrm{~d} x \\
& =\frac{1}{\ln 2} \int_{0}^{\infty} \frac{1-\operatorname{Pr}\left(\frac{Z_{k^{*}} Y_{k^{*}}}{Z_{k^{*}}+Y_{k^{*}}+1}<\frac{x}{a_{1}-a_{2} x}\right)}{1+x} \mathrm{~d} x \\
& =\frac{1}{\ln 2} \int_{0}^{\frac{a_{1}}{a_{2}}} \frac{1-\operatorname{Pr}\left(\frac{Z_{k^{*}} Y_{k^{*}}}{Z_{k^{*}+Y_{k^{*} 1}+1}}<\frac{x}{a_{1}-a_{2} x}\right)}{1+x} \mathrm{~d} x \\
& +\frac{1}{\ln 2} \int_{\frac{a_{1}}{a_{2}}}^{\infty} \frac{1-\operatorname{Pr}\left(\frac{Z_{k^{*}} Y_{k^{*} 1}}{Z_{k^{*}}+Y_{k^{*} 1}+1}>\frac{x}{a_{1}-a_{2} x}\right)}{1+x} \mathrm{~d} x \\
& =\frac{1}{\ln 2} \int_{0}^{\frac{a_{1}}{a_{2}}} \frac{1-\operatorname{Pr}\left(\frac{Z_{k^{*}} Y_{k^{*} 1}}{Z_{k^{*}+}+Y_{k^{*} 1}+1}<\frac{x}{a_{1}-a_{2} x}\right)}{1+x} \mathrm{~d} x \\
& \stackrel{\text { (h) }}{<} \frac{1}{\ln 2} \int_{0}^{\frac{a_{1}}{a_{2}}} \frac{1-\operatorname{Pr}\left(\frac{Z_{k^{*}} Y_{k^{*} 1}}{Z_{k^{*}+Y_{k^{*}}}}<\frac{x}{a_{1}-a_{2} x}\right)}{1+x} \mathrm{~d} x \\
& \stackrel{(i)}{\approx} \frac{1}{\ln 2} \int_{0}^{\frac{a_{1}}{a_{2}}} \frac{1-\operatorname{Pr}\left(\min \left\{Z_{k^{*}}, Y_{k^{*} 1}\right\}<\frac{x}{a_{1}-a_{2} x}\right)}{1+x} \mathrm{~d} x \\
& \stackrel{(j)}{=} \frac{1}{\ln 2} \int_{0}^{\frac{a_{1}}{a_{2}}} \frac{\left(1-F_{Z}\left(\frac{x}{a_{1}-a_{2} x}\right)^{K}\right)\left(1-F_{Y_{1}}\left(\frac{x}{a_{1}-a_{2} x}\right)\right)}{1+x} \mathrm{~d} x
\end{aligned}
$$

where step $(h)$ follows from neglecting the constant 1 in the denominator of the left-hand side in the $\operatorname{Pr}(\cdot)$ operator, as this yields a good approximation at medium-to-high SNR; step (i) follows from the known result that the minimum between two values is an upper bound for their harmonic mean, which also becomes increasingly tight at medium-to-high SNR; and step $(j)$ follows under the consideration of i.i.d. fading channels. In the above expression, $F_{Z}(\cdot)$ is the CDF of the RV $Z$ given as in (40) and $F_{Y 1}\left(y_{1}\right)=1-\exp \left(-y_{1} / \bar{\gamma}_{\mathrm{RD}_{1}}\right)$. Therefore, after solving the integral in (43) with the use of [35], a closed-form approximation for the ergodic capacity of the weak user $D_{1}$ can be obtained as in (32).

\section{APPENDIX D}

\section{PROOF OF PROPOSITION 8}

By following a similar procedure as in the proof of Proposition 7 , we derive the ergodic capacity for strong user $D_{2}$. By substituing (12) into (37), the ergodic capacity for $\mathrm{D}_{2}$ can be developed as

$$
\begin{aligned}
\bar{C}_{2} & =\frac{1}{\ln 2} \int_{0}^{\infty} \frac{1-\operatorname{Pr}\left(\frac{a_{2} Z_{k^{*} Y_{k^{*} 2}}}{Z_{k^{*}+Y_{k^{*}}+1}}<x\right)}{1+x} \mathrm{~d} x \\
& =\frac{1}{\ln 2} \int_{0}^{\infty} \frac{1-\operatorname{Pr}\left(\frac{Z_{k^{*} Y_{k^{*} 2}}}{Z_{k^{*}+Y_{k^{*}}+1}}<\frac{x}{a 2}\right)}{1+x} \mathrm{~d} x \\
& \stackrel{(h)}{<} \frac{1}{\ln 2} \int_{0}^{\infty} \frac{1-\operatorname{Pr}\left(\frac{Z_{k^{*} Y_{k^{*}}}}{Z_{k^{*}+Y_{k^{*} 2}}}<\frac{x}{a 2}\right)}{1+x} \mathrm{~d} x
\end{aligned}
$$

$$
\begin{aligned}
& \stackrel{(i)}{\approx} \frac{1}{\ln 2} \int_{0}^{\infty} \frac{1-\operatorname{Pr}\left(\min \left\{Z_{k^{*}}, Y_{k^{*} 2}\right\}<\frac{x}{a_{2}}\right)}{1+x} \mathrm{~d} x \\
& \stackrel{(j)}{=} \frac{1}{\ln 2} \int_{0}^{\infty} \frac{\left(1-F_{Z}\left(\frac{x}{a_{2}}\right)^{K}\right)\left(1-F_{Y_{2}}\left(\frac{x}{a_{2}}\right)\right)}{1+x} \mathrm{~d} x,
\end{aligned}
$$

from which, after solving the integral in (44), a closed-form approximation for the ergodic capacity of the strong user $\mathrm{D}_{2}$ can be attained as in (33).

\section{REFERENCES}

[1] H. Tullberg, P. Popovski, Z. Li, M. A. Uusitalo, A. Hoglund, O. Bulakci, M. Fallgren, and J. F. Monserrat, "The METIS 5G system concept: Meeting the 5G requirements," IEEE Commun. Mag., vol. 54, no. 12, pp. 132-139, Dec. 2016.

[2] Z. Ding, X. Lei, G. K. Karagiannidis, R. Schober, J. Yuan and V. K. Bhargava, "A survey on non-orthogonal multiple access for $5 \mathrm{G}$ networks: Research challenges and future trends," IEEE J. Sel. Areas Commun., vol. 35, no. 10, pp. 2181-2195, Oct. 2017.

[3] Y. Liu, Z. Qin, M. Elkashlan, Z. Ding, A. Nallanathan, and L. Hanzo, "Nonorthogonal multiple access for 5G and beyond," Proc. IEEE, vol. 105, no. 12, pp. 2347-2381, Dec. 2017.

[4] Y. Saito, Y. Kishiyama, A. Benjebbour, T. Nakamura, A. Li, and K. Higuchi, "Non-orthogonal multiple access (NOMA) for cellular future radio access," in Proc. IEEE 77th Veh. Tech. Conf. (VTC-Spring), pp. 15, Jun 2013.

[5] S. M. R. Islam, N. Avazov, O. A. Dobre and K. s. Kwak, "Power-domain non-orthogonal multiple access (NOMA) in 5G systems: Potentials and challenges," in IEEE Commun. Surveys Tuts., vol. 19, no. 2, pp. 721742, Oct. 2016.

[6] J. Laneman, D. Tse, and G. W. Wornell, "Cooperative diversity in wireless networks: Efficient protocols and outage behavior," IEEE Trans. Inf. Theory, vol. 50, no. 12, pp. 3062-3080, Dec. 2004.

[7] E. E. Benitez Olivo, D. P. Moya Osorio, D. B. da Costa, and J. C. S. Santos Filho, "Multiuser incremental decode-and-forward relaying under outdated channel estimates," Electronics Lett., vol. 51, no. 4, pp. 369371, Feb. 2015.

[8] D. P. Moya Osorio, E. E. Benitez Olivo, H. Alves, J. C. S. Santos Filho, and M. Latva-aho, "An adaptive transmission scheme for amplify-andforward relaying networks," IEEE Trans. Commun., vol. 65, no. 1, pp. 66-78, Jan. 2017.

[9] L. Zhang, J. Liu, M. Xiao, G. Wu, Y. Liang, and S. Li, "Performance analysis and optimization in downlink NOMA systems with cooperative full-duplex relaying," IEEE J. Sel. Areas Commun., vol. 35, no. 10, pp. 2398-2412, Oct. 2017.

[10] J. B. Kim and I. H. Lee, "Non-orthogonal multiple access in coordinated direct and relay transmission," IEEE Commun. Lett., vol. 19, no. 11, pp. 2037-2040, Nov. 2015.

[11] C. Zhong and Z. Zhang, "Non-orthogonal multiple access with cooperative full-duplex relaying," IEEE Commun. Lett., vol. 20, no. 12, pp. 2478-2481, Dec. 2016.

[12] R. Tang, J. Cheng, and Z. Cao, "Contract-based incentive mechanism for cooperative NOMA systems," IEEE Commun. Lett., vol. 23, no. 1, pp. 172-175, Jan. 2019.

[13] J. Men, J. Ge, and C. Zhang, "Performance analysis of non-orthogonal multiple access for relaying networks over Nakagami- $m$ fading channels," IEEE Trans. Veh. Technol., vol. 66, no. 2, pp. 1200-1208, Feb. 2017.

[14] S. Lee, D. B. da Costa, Q. T. Vien, T. Q. Duong, and R. T. de Sousa, "Non-orthogonal multiple access schemes with partial relay selection," IET Commun., vol. 11, no. 6, pp. 846-854, Apr. 2017.

[15] D. Deng, L. Fan, X. Lei, W. Tan, and D. Xie, "Joint user and relay selection for cooperative NOMA networks," IEEE Access., vol. 5, pp. 20220-20227, Sep 2017.

[16] Y. Li, Y. Li, X. Chu, Y. Ye and H. Zhang, "Performance analysis of relay selection in cooperative NOMA networks," IEEE Commun. Lett., vol. 23, no. 4, pp. 760-763, Apr. 2019.

[17] N. Guo, J. Ge, C. Zhang, Q. Bu and P. Tian, "Non-orthogonal multiple access in full-duplex relaying system with Nakagami- $m$ fading," IET Commun., vol. 13, no. 3, pp. 271-280, Feb. 2019.

[18] Z. Yang, Z. Ding, Y. Wu and P. Fan, "Novel relay selection strategies for cooperative NOMA," IEEE Trans. Veh. Technol., vol. 66, no. 11, pp. 10114-10123, Nov. 2017. 
[19] P. Xu, Z. Yang, Z. Ding and Z. Zhang, "Optimal relay selection schemes for cooperative NOMA," IEEE Trans. Veh. Technol., vol. 67, no. 8, pp. 7851-7855, Aug. 2018.

[20] Q. Y. Liau, C. Y. Leow, and Z. Ding, "Amplify-and-forward virtual fullduplex relaying-based cooperative NOMA," IEEE Wireless Commun. Lett., vol. 7, no. 3, pp. 464-467, Jun. 2018.

[21] R. Tang, J. Cheng, and Z. Cao, "Energy-efficient power allocation for cooperative NOMA systems with IBFD-enabled two-way cognitive transmission," IEEE Commun. Lett., vol. 23, no. 6, pp. 1101-1104, Jun. 2019.

[22] X. Wang, M. Jia, I. W. Ho, Q. Guo, and F. C. M. Lau, "Exploiting full-duplex two-way relay cooperative non-orthogonal multiple access," IEEE Trans. Commun., vol. 67, no. 4, pp. 2716-2729, Apr. 2019.

[23] M. Duarte, C. Dick, and A. Sabharwal, "Experiment-driven characterization of full-duplex wireless systems," IEEE Trans. Wireless Commun., vol. 11 , no. 12 , pp. 4296-4307, Dec. 2012.

[24] M. Heino et al., "Recent advances in antenna design and interference cancellation algorithms for in-band full duplex relays," IEEE Commun. Mag., vol. 53, no. 5, pp. 91-101, May 2015.

[25] T. Riihonen, S. Werner and R. Wichman, "Hybrid full-duplex/halfduplex relaying with transmit power adaptation," IEEE Trans. Wireless Commun., vol. 10, no. 9, pp. 3074-085, Sep. 2011.

[26] D. P. Moya Osorio, E. E. Benitez Olivo, H. Alves, J. C. S. Santos Filho, and M. Latva-aho, "Exploiting the direct link in full-duplex amplify-andforward relaying networks," IEEE Sig. Process. Lett., vol. 22, no. 10, pp. 1766-1770, Oct. 2015.

[27] I. Krikidis, H. Suraweera, P. Smith, and C. Yuen, "Full-duplex relay selection for amplify-and-forward cooperative networks," IEEE Trans. Wireless Commun., vol. 11, no. 12, pp. 4381-4393, Dec. 2012.

[28] Z. Zhang, H. Sun, and R. Q. Hu, "Downlink and uplink non-orthogonal multiple access in a dense wireless network," IEEE J. Sel. Areas Commun., vol. 35, no. 12, pp. 2771-2784, Dec. 2017.

[29] F. Liu, P. Mahonen, and M. Petrova, "Proportional fairness-based power allocation and user set selection for downlink NOMA systems," in Proc. IEEE Int. Conf. Commun. (ICC), pp. 1-6, May 2016.

[30] A. Tregancini, C. H. M. Lima, E. E. Benitez Olivo, and H. Alves, "Performance analysis framework for NOMA systems over non-identical Nakagami-m fading channels," in Proc. 16th Int. Symp. Wireless Commun. Syst. (ISWCS), Oulu, Finland, 2019, pp. 150-154.

[31] E. Dosti, M. Shehab, H. Alves, and M. Latva-aho, "On the performance of non-orthogonal multiple access in the finite blocklength regime," $\mathrm{Ad}$ Hoc Networks, Oct. 2018.

[32] H. Qu, R. Tang, J. Zhao, and Z. Cao, "Performance tradeoff between user fairness and energy conservation in downlink NOMA systems," in Proc. IEEE ICC Workshops, Kansas City, MO, USA, 2018, pp. 1-6.

[33] M. Abramowitz and I. Stegun, Handbook of Mathematical Functions with Formulas, Graphs, and Mathematical Tables. New York, NY, USA: Dover, 1972.

[34] A. Papoulis, Probability, Random Variables, and Stochastic Processes, 4th ed. New York, NY, USA: McGraw-Hill, 2002.

[35] Wolfram Research, Inc. (2019), "The Mathematical Functions Site: Exponential Function," [Online]. Avaliable: http://functions.wolfram.com/ 01.03.21.0060.01

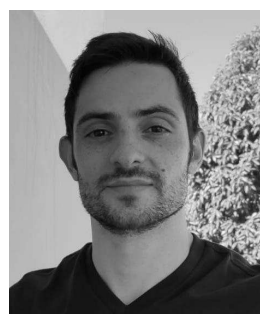

Anderson Tregancini received the B.Sc. degree in Telecommunication Engineering from São Paulo State University (UNESP), Campus of São João da Boa Vista, São João da Boa Vista, Brazil, in 2018, and is currently working toward the M.Sc. degree in Electrical Engineering at UNESP. His research interests are related to wireless communications with emphasis on the performance analysis of NOMAbased cooperative networks.

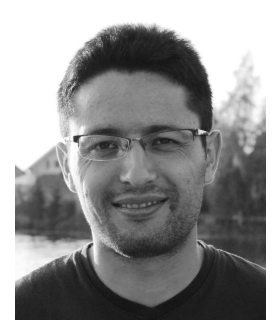

Edgar Eduardo Benitez Olivo (M'16) received the B.Sc. degree in Electronics and Telecommunications Engineering from the Armed Forces UniversityESPE, Ecuador, in 2008, and the M.Sc. and Ph.D. degrees in Electrical Engineering from the University of Campinas, Brazil, in 2011 and 2015, respectively. In 2014, he held a visiting researcher position with the Centre for Wireless Communications, University of Oulu, Finland. Since 2016, he has been with São Paulo State University (UNESP), Campus of São Jõao da Boa Vista, Brazil, where he is currently an Assistant Professor. He has served as a reviewer for many IEEE and non-IEEE journals and has been involved as a TPC member in several conferences. His research interests lie in the area of wireless communications, with a current focus on emerging technologies towards $5 \mathrm{G}$ wireless networks.

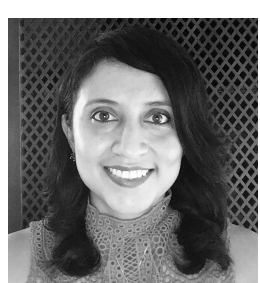

Diana Pamela Moya Osorio (M'16) was born in Quito, Ecuador. She received the B.Sc. degree in electronics and telecommunications engineering from the Armed Forces University-ESPE, Sangolquí, Ecuador, in 2008, and the M.Sc. and D.Sc. degrees in electrical engineering with emphasis on telecommunications and telematics from the University of Campinas - UNICAMP, Campinas, Brazil, in 2011 and 2015, respectively. In 2018, she was a Visiting Researcher at the Centre for Wireless Communications (CWC) at University of Oulu, Finland, during one year. Since 2015, she is acting as an Assistant Professor with the Department of Electrical Engineering, Federal University of São Carlos (UFSCar), São Carlos, Brazil. Since 2020, she is also acting as Senior Researcher at CWC, University of Oulu. She has served as TPC and reviewer for several journals and conferences. Her research interests include wireless communications in general, PHY security, machine-type communications, and UAV-based communications.

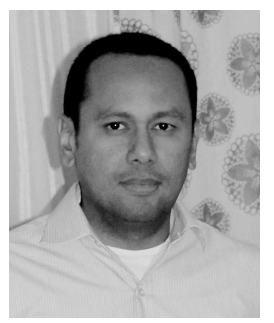

Carlos H. M. de Lima received the B.Sc. and M.Sc. in Electrical Engineering from the Federal University of Ceará (UFC), Brazil in 2002 and 2004, respectively. In 2013, he received the Dr.Sc (Tech) degree in electrical engineering from the University of Oulu, Finland. From 2000 to 2005, he worked as research scientist in the Wireless Telecommunication Research Group (GTEL), Brazil. In 2005 he was a visiting researcher in the Ericsson Research Center, Luleå, Sweden. In 2006, he worked at the Nokia Institute of Technology (INdT), Brazil. From 2014 to 2018, he worked as an assistant professor at the Telecommunications Engineering Department, São Paulo State University (UNESP), Brazil. Currently, he is a senior research fellow with the Centre for Wireless Communications (CWC), Department of Communications and Engineering (DCE), University of Oulu, Finland. His research interests are statistical signal processing, data fusion and analytics, stochastic geometry and probabilistic graphical models.

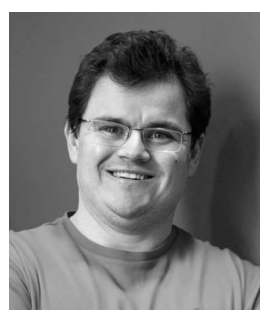

Hirley Alves (S'11-M'15) received the B.Sc. and M.Sc. degrees from the Federal University of Technology-Paraná (UTFPR), Brazil, in 2010 and 2011, respectively, both in electrical engineering, and the dual D.Sc. degree from the University of Oulu and UTFPR, in 2015. In 2017, he was an Adjunct Professor in machine-type wireless communications with the Centre for Wireless Communications (CWC), University of Oulu, Oulu, Finland. In 2019, he joined CWC as an Assistant Professor and is currently the Head of the Machine-type Wireless Communications Group. He is actively working on massive connectivity and ultra-reliable low latency communications for future wireless networks, $5 \mathrm{~GB}$ and 6G, full-duplex communications, and physical-layer security. He leads the URLLC activities for the 6G Flagship Program. He is a co-recipient of the 2017 IEEE International Symposium on Wireless Communications and Systems (ISWCS) Best Student Paper Award, and 2019 IEEE European Conference on Networks and Communications (EuCNC) Best Student Paper Award and a co-recipient of the 2016 Research Award from the Cuban Academy of Sciences. He has been the organizer, chair, and TPC and tutorial lecturer for several renowned international conferences. He is the General Chair of the ISWCS'2019 and the General Co-Chair of the 1st 6G Summit, Levi 2019, and ISWCS 2020. 